\title{
Algunas CONTRIBUCIONES A LA COMPRENSIÓN DE LA TRANSICIÓN Paracas-Nasca, Samaca, valle bajo de Ica
}

\author{
Lauren Cadwallader ${ }^{\text {a }}$ \\ Susana Arce Torres ${ }^{\mathrm{b}}$ \\ Alexander G. Pullen ${ }^{\mathrm{C}}$ \\ David G. Beresford-Jones ${ }^{\mathrm{d}}$
}

\begin{abstract}
Resumen
Hasta hace poco, la transición entre el final de la época Paracas (380-260 a.C.) y la subsiguiente Nasca Inicial (260 a.C.-80 d.C.) de la costa sur del Perú, se ha estudiado principalmente a través de los restos funerarios y los análisis del patrón de asentamiento. Sin embargo, si intentamos entender los mecanismos socio-culturales y politicos que subyacen a estos cambios grandes, necesitamos un mejor conocimiento de los detalles de la vida cotidiana de aquella gente. Con información de recientes investigaciones, aqui pretendemos contribuir al entendimiento de la ocupación semipermanente de un sitio Ocucaje 9/10-Nasca 1, (Samaca 1004) en la Cuenca de Samaca, en la parte baja del valle de Ica. El sitio se encuentra sobre la pampa, en el borde de la cuenca, aproximadamente a 80 metros por encima de la planicie aluvial del actual río Ica y distante de cualquier fuente de agua. El sitio está asociado con una serie de geoglifos trapezoidales marcados en la superficie de esta pampa.
\end{abstract}

A través de la evidencia —cerámica, litica, zoológica, botánica y funeraria - recolectados sistemáticamente de la superficie, propenemos varias hipótesis sobre qué tipo de sitio fue Samaca 1004 y porqué se ubicó en ese lugar.

Palabras clave: Transición Paracas-Nasca; geoglifos; dieta humana, análisis de isótopos, camélidos domesticados

\section{Abstract \\ Contributions to the understanding of the Paracas-Nasca transition from Samaca. lower Ica Valley}

Until recently the transition between the Paracas (380 - 260 B.C.) and subsequent Initial Nasca (260 B.C. - A.D. 80) epochs on the south coast of Peru was understood primarily through mortuary remains, and settlement pattern analysis. If we are to understand properly the socio-cultural and political mechanisms that underlay this period of great change, however, we need a better understanding of the quotidian details of the lives of those people who made and experienced them. We seek here to contribute to that understanding by reporting recent investigations of a semi-permanent Ocucaje

${ }^{a}$ Office of Scholarly Communication, Cambridge University Library

Correo electrónico: lc340@cam.ac.uk

${ }^{\text {b }}$ Museo Regional de Ica (DD-Ica, Ministerio de Cultura)

Correo electrónico: susarceto@yahoo.com

c Pre-Construct Archaeology

Correo electrónico: agp27@hotmail.com

d McDonald Institute for Archaeological Research, University of Cambridge Correo electrónico: dgb27@cam.ac.uk 
9/10 - Nasca 1 occupation site in the Samaca Basin, lower Ica Valley ('Samaca 1004'). The site lies on the pampa at the edge of the basin, approximately $80 \mathrm{~m}$ above the current Rio Ica floodplain and distant from any source of water. The site is associated with a series of trapezoid geoglyphs marked on the surface of this pampa.

Using ceramic, lithic, faunal, botanical and mortuary evidence gathered from field-walking survey, we propose various hypotheses for what kind of site Samaca 1004 was, and why it was situated where it was.

Keywords: Paracas-Nasca transition; geoglyphs; human diet, isotope analysis, domesticated camelids.

\section{Introducción}

El fin de alrededor de ocho siglos de influencia del Horizonte Temprano en la Costa Sur del Perú y la transición a la época Nasca, se ha considerado durante mucho tiempo como una época de grandes cambios sociales y políticos. La historia cultural de esta época fue definida por Menzel y colegas (1964) según una secuencia de cerámica Ocucaje, las fases finales de las cuales se conocen como «Paracas». En el valle de Ica, cambios en los patrones de asentamiento Paracas, en las técnicas de construcción, en la iconografía, así como en las técnicas de elaboración de los bienes de alta calidad y su uso exclusivo por la elite, sugieren una significativa agitación política y social en las fases Ocucaje 9 y 10 (Menzel et al. 1964; Massey 1991; Cook 1999; DeLeonardis 2005). Esta imagen ha sido aumentada por nuevas investigaciones sobre este período crítico, fuera de Ica en el valle de Chincha (Canziani 2103; Stanish et al. 2014; Tantaleán et al. 2013, 2016) y en el valle bajo de Ica (Bachir Bacha y Llanos 2013; Bachir Bacha 2017).

Para comprender debidamente los mecanismos socioculturales y políticos que subyacen a estos cambios, también necesitamos un mejor conocimiento de los detalles de la vida cotidiana de quienes los experimentaron. En el valle de Ica los principales sitios de Paracas y Nasca Inicial están ubicados en el valle medio en Cerrillos y en el valle bajo en Callango y Ocucaje; todos ellos con importantes áreas propicias para la agricultura.

Aquí ofrecemos una contribución modesta a través de investigaciones en un sitio Paracas Tardío recientemente registrado, en la pampa por encima de la cuenca de Samaca, valle bajo de Ica, con evidencias que sugieren que pudo haber tenido algún componente doméstico, además de entierros. El sitio está, además, muy próximo a un conjunto de geoglifos trapezoidales en la pampa, posiblemente contemporáneo. La cuenca de Samaca es una región de potencial agrícola limitado, al parecer, debido a la naturaleza efímera del río Ica y el área limitada de tierra potencialmente cultivable en el valle. Sin embargo, la cuenca de Samaca está ventajosamente ubicada cerca de las ecologías de la costa del Pacífico y sus lomas adyacentes, cada cual con importantes recursos potenciales.

\section{Samaca 1004: un sitio Paracas Transicional}

\subsection{Ubicación}

El sitio Samaca 1004 (en lo sucesivo 1004), se encuentra en lo alto de las laderas occidentales de la cuenca Samaca, casi encima de la pampa del Tablazo de Ica, y aproximadamente a 80 metros sobre el nivel del río actual. Hoy este parece un sitio improbable para cualquier forma de asentamiento, dado que está expuesto a la intensa radiación solar de la región y a un régimen de vientos extraordinarios, con una velocidad promedio de viento continuo de 30 kilómetros por hora y ráfagas frecuentes por encima de 115 kilómetros por hora (Beresford-Jones 2011). El sitio ocupa un área relativamente plana entre las laderas de cerros que conducen a la pampa, con cerros más pequeńos. Partes de él han sido saqueadas disturbando entierros humanos. Una serie de geoglifos y paravientos asociados se encuentran muy cerca, en las pampas ubicadas justo por encima de 1004 (Fig. 1). 

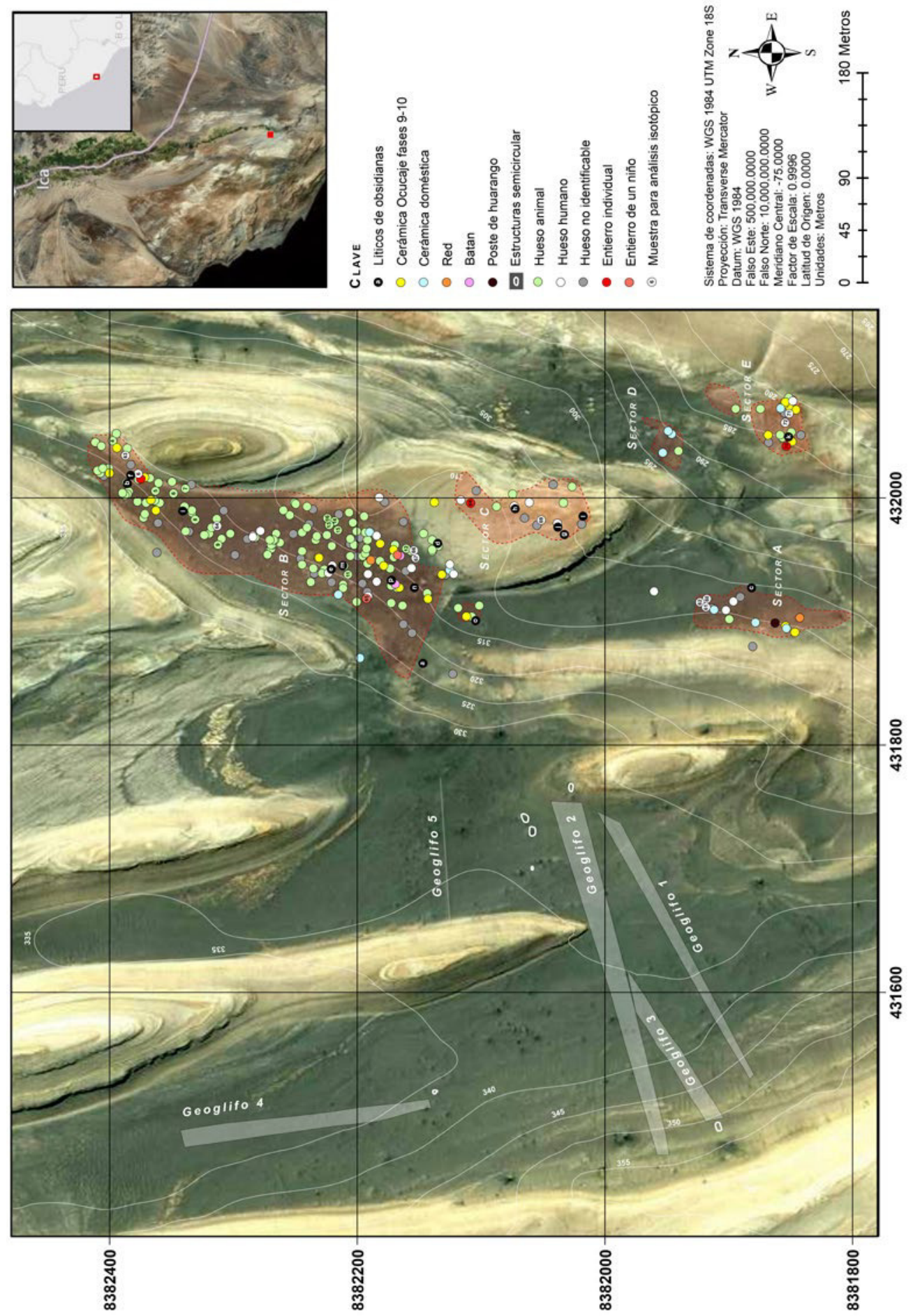

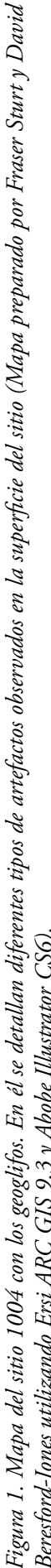


El sitio fue explorado con GPS diferencial y caminado sistemáticamente en transectos a fin de recolectar restos humanos de su superficie para la investigación isotópica, registrando a la vez la cultura material asociada a ellos (Cadwallader 2013; Cadwallader et al. 2018). La osteología y patología de los restos humanos de 1004 fueron estudiadas en detalle en el campo y las muestras tomadas fueron analizadas posteriormente en la Universidad de Cambridge.

El sitio 1004 abarca un área de aproximadamente 700 metros N-S y 250 metros E-O. Fue dividido en cinco sectores. Los sectores A, D y E son áreas discretas en cuanto a materiales arqueológicos, mientras que los sectores $\mathrm{B}$ y $\mathrm{C}$, divididos en dos por sus características topográficas y geológicas subyacentes, presentan la dispersión más densa de restos arqueológicos en su superficie.

Sector A. Es el área arqueológica en el extremo suroeste de todo el sitio, parecida a un óvalo de forma alargada orientado sobre el eje Norte-Sur y de un tamaño de 126 por 35 metros (N-S por E-O). Se encuentra en la base oriental de la pendiente que sube hasta la pampa.

Sector B. Es el sector más grande, 300 metros N-S por 80 metros E-O. La forma de este sector sigue más o menos la forma de este lado del valle.

Sector $C$. Este sector está ubicado en la cima de un promontorio, que, en su extremo sur, se eleva por encima de los sectores A, D y E, descendiendo por el Norte al nivel del Sector B. Aunque los restos arqueológicos y el saqueo son continuos entre este sector y el $\mathrm{B}$, la división entre ambos fue porque hay una línea de cambio geológico natural por la que hay superficies con arena eólica en el Sector $\mathrm{B}$, mientras que el Sector $\mathrm{C}$ presenta muy poca arena cobre caliza con piedras pequeñas. Las dimensiones de este sector son 90 metros N-S por 20 metros E-O.

Sector D. Ubicado al sureste de la base del promontorio del Sector C. Al Sur se encuentra el Sector E y al Este el suelo firme declina hacia el valle. Este Sector tiene forma irregular reflejando la actividad de los saqueadores (huaqueros). Su área principal mide aproximadamente 25 metros N-S por 35 metros E-O.

Sector E. Ubicado al Sur del Sector D, casi al mismo nivel que los Sectores A, B y D. Su superficie está compuesta por dos áreas adyacentes ovaladas, orientadas sobre el eje noreste-suroeste, de aproximadamente 54 por 38 metros y 32 por 21 metros, respectivamente.

La apariencia de huesos blanqueados por el sol y enterrados en la arena dentro de los pozos de «huaqueros» sugiere que 1004 fue saqueada hace muchos años. En efecto, el hijo de Seferino Galindo, «huaquero» responsable de gran parte de su saqueo, informa que fue saqueado en varias ocasiones durante unos 35 años (comunicación personal). El tamaño y la profundidad de los hoyos de los saqueadores indica, a diferencia de partes más accesibles de estas cuencas que fueron saqueadas con maquinaria, que 1004 fue saqueada a mano. Estos pozos varían en tamaño, encontrándose el más grande (2 metros de diámetro aprox.) en el Sector B. Hubo mucho menos actividad de huaqueo en el Sector D y pocos materiales arqueológicos en su superficie. Aparentemente, este sector no fue disturbado en el mismo grado que los otros, probablemente porque hubo menos restos que saquear.

\subsection{Cultura material}

En la superficie de 1004 se observó tanto cerámica decorada como utilitaria; recogiéndose solo cerámica diagnóstica con el propósito de ubicar cronológicamente e intentar caracterizar el sitio. Entre esta se recogió una variedad de diseños, colores y formas de vasijas, cuya gran mayoría corresponde a las fases Ocucaje 9 y 10 (Menzel et al. 1964).

Cerca de una de las concentraciones de restos humanos en el Sector C, se observaron muchos fragmentos de ollas grandes, vasijas de cocina en las formas típicas de las de las fases finales de Ocucaje (v.g., Menzel et al. 1964: 344). Estas podrían haber sido utilizadas para el entierro de infantes, aunque no se observaron evidencias directas de ello. Entre los fragmentos de cerámica diagnóstica de Ocucaje 9 y 10 y Nasca 1 que se recolectaron, hay cerámica negra bruñida con diseños incisos; diseños incisos y pintados con resina; diseños en negativo y fragmentos de antaras (cf. Menzel et al. 1964: 369-382; figs. 2 a-c y f). 

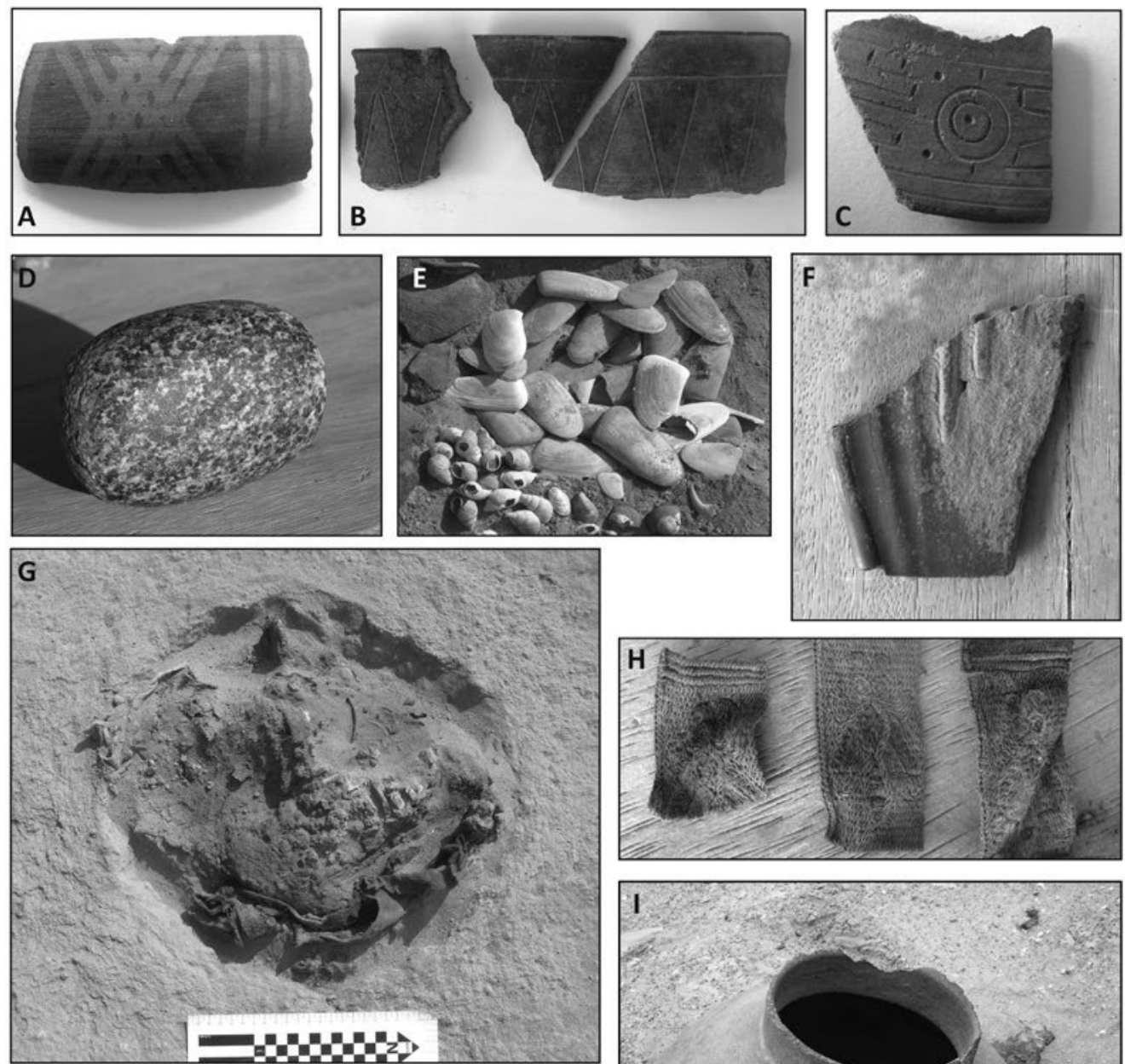

De arriba a abajo:

A-C Fragmentos de cerámica, fases Ocucaje 9/10 (cf. Menzel et al. 1964):

$A$ - diseños en negativo (sector $A$ );

$B$ - cerámica negra con diseños incisos (sector $B$ ); $C$ - cerámica incisa con diseños de resina post-cocción (sector B).

D $\quad$ Mano de mortero en granito verde (sector B).

E Restos de moluscos marinos y terrestres (sector B).

F $\quad$ Fragmento de antara (sector B).

$G \quad$ Entierro de un niño en hoyo circular (sector B).

H Fragmento de tela bordada 'Paracas' (sector B).

I Olla, Ocucaje tardio (sector B).
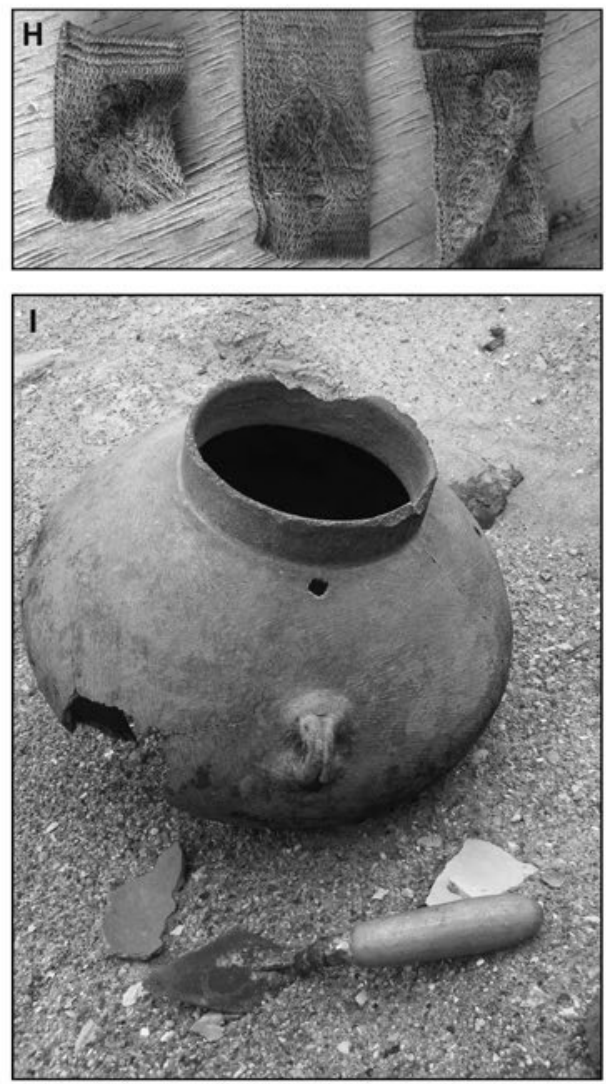

Figura 2. Artefactos observados en la superficie de 1004 (Fotografias de Lauren Cadwallader y David Beresford-Jones). 
En el Sector B, algunos fragmentos cerámicos decorados del estilo Nasca, fases $2 / 3$, fueron hallados en la superficie del extremo norte de ese sector (por lo tanto del sitio), estimándose que no representan una presencia importante.

También se observaron fragmentos textiles llanos y decorados entre los restos de cultura material. Aparentemente, los restos humanos fueron envueltos con tejidos llanos de algodón. Uno de ellos había sido teñido de rojo, pero fue difícil identificar el color original de otros ejemplos debido a la degradación del color por la intemperie. Se recuperaron además, fragmentos de greca bordada cosida a un textil llano Paracas, en asociación con uno de los individuos muestreados (Fig. 2h, cf. Stone-Miller 1992: 91).

Se registraron numerosos fragmentos de redes de algodón, probablemente para la pesca. También se registraron en el sitio 1004 dos batanes grandes, así como también manos de moler (Fig. 2d); estos artefactos no son fáciles de transportar, sugiriendo que invirtieron cierto grado de trabajo al traerlos a este sitio. Otros restos de artefactos son los fragmentos de muchas vasijas domésticas —algunos de los cuales presentaron hollín en el exterior como evidencia de uso (Fig. 2i) —, y un implemento agrícola de madera. No hemos visto ninguna evidencia directa de estructuras arquitectónicas en 1004, aunque por supuesto, el sitio no ha sido excavado. Hay sin embargo, algunas sugerencias de materiales estructurales como un poste corto de huarango y grupos de cańas, entre los restos del Sector B, que podrían haber sido usados para techos.

Por otro lado, en la superficie de 1004 se recuperaron una serie de herramientas trabajadas bifacialmente $(n=34)$, pequeñas lascas $(n=11)$ y un pequeño núcleo de obsidiana (Fig. 3). La mayoría de las herramientas muestra evidencias de haber llevado mango. Una punta y una lasca fueron hechas en cuarcita. Otra punta bifacial con lados irregulares fue elaborada en cuarzo marrón. La obsidiana fue utilizada durante todo el Horizonte Temprano para la fabricación de herramientas y armas, y se ha encontrado en contextos rituales y domésticos (Engel 1981; DeLeonardis y Glascock 2013; Bachir Bacha y Llanos 2013).

Por lo general, las puntas de 1004 son simétricas y triangulares (es decir con los bordes laterales rectos), o en forma de hoja (es decir con los bordes laterales convexos). Las bases de estas herramientas suelen ser rectas o ligeramente cóncavas (ver los tipos I-1-4/5/7 y X-1-5/7 en Engel 1981: 10), aunque hay excepciones. Además, se recuperaron dos artefactos grandes de obsidiana (Figs. $3 \mathrm{~b}$ y $3 \mathrm{~g}$ ). Uno de estos, con un borde retocado, posiblemente un cuchillo, se encontró envuelto con dos pequeñas cuentas en un fragmento textil, a manera de una pequeña bolsa de algodón con cordón atado, pero muy deteriorados. La otra pieza bifacial fue hallada también en la superficie muy cerca a la anterior.

Los bifaciales de 1004 son similares a algunos del sitio Ocucaje 9 de Ánimas Altas, descritos por Burger (2007: figs. 2 y 3) y Bachir Bacha y Llanos (2013: fig. 26). Los artefactos de obsidiana recolectados de 1004, muestran poca variedad, al tratarse probablemente solo de puntas de proyectil y cuchillos. Burger (2007) sostiene que algunos de los bifaciales de Ánimas Altas fueron proyectiles con mango usados en el ataque, la guerra y la caza. Curiosamente el análisis de una punta de obsidiana de 1004 mediante microscopio electrónico de barrido (SEM), acoplado a un analizador elemental, detectó adherencias de sangre en su superficie ${ }^{1}$ (Fig. 3f).

Las grandes piezas de obsidiana con la especie de bolsa de tela y cuentas probablemente proceden de un contexto funerario, debido a su cercanía a restos óseo humanos. Además de su potencial función como cuchillos, en caso de necesidad, estos grandes bifaciales habrían servido como una fuente de lascas de obsidiana para la fabricación de herramientas. El único núcleo de obsidiana observado también pudo ser una ofrenda funeraria. Hay varios ejemplos confiables de herramientas de obsidiana o armas que fueron depositadas como ofrendas en cementerios del Horizonte Temprano (Engel 1991; DeLeonardis y Glascock 2013). 

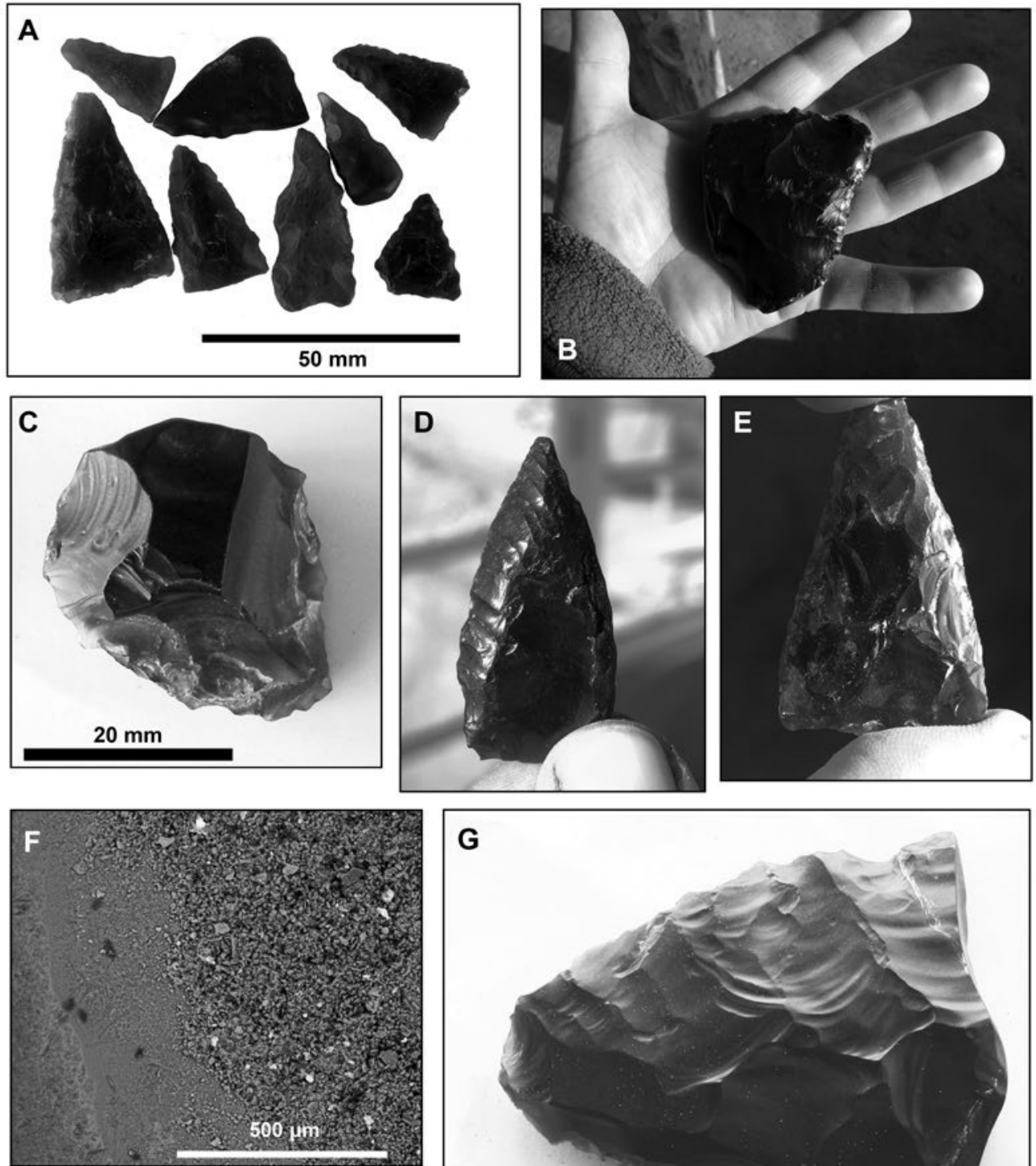

De arriba a abajo:

A Selección de puntas de varias formas, algunas pulidas por el viento.

B Cuchillo bifacial hallado envuelto en fragmento textil.

C Pequeño núcleo.

D Punta bifacial con lados convexos y base plana. Evidencia de

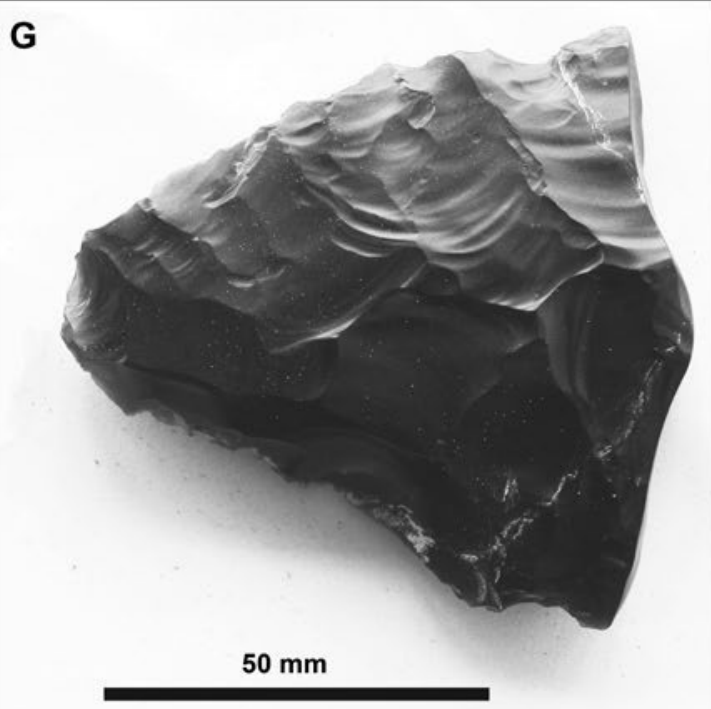
enmangamiento.

E Punta bifacial con lados rectilineos $y$ base plana.

F $\quad$ Superficie del punta bajo microscopio electrónico que muestra restos de sangre.

$G \quad$ Cuchillo bifacial encontrado cerca de la bolsa.

Figura 3. Selección de algunos de los artefactos de obsidiana observados en la superficie de 1004. Fotos A-E y G por Alexander Pullen (Foto F: cortesía de la Dra. Gladys Ocharán, Laboratorio MYAP, www.myapcompany.com) 
Estudios de abastecimiento de obsidiana sobre puntas excavadas de varios sitios en el valle de Ica indican que la fuente más probable de la obsidiana de 1004 estuvo distante a $225 \mathrm{~km}$, en Quispisisa (Burger y Glascock 2000; Burger 2007; Tripcevich y Contreras 2011). Aparte de las lascas y un único núcleo de obsidiana, hay pocas evidencias en la superficie de 1004 de la manufactura de artefactos de obsidiana en el sitio. Hubo poca evidencia de desechos de talla de obsidiana en Ánimas Altas, aunque al parecer, restos de obsidiana de todas las fases de producción son visibles en la superficie de los sitios Paracas en el sector Fundo Santa Rosa del Valle Bajo de Ica (Burger 2007; DeLeonardis y Glascock 2013). Es probable que para la fabricación de herramientas en contextos domésticos del Horizonte Temprano se hayan usado materias primas locales, preferentemente.

Algunas de las puntas de 1004, con las huellas de trabajo casi completamente borradas por la abrasión del viento, deben de haber permanecido durante un largo período en la superficie del desierto (Fig. 3a). Sin embargo, la mayoría de los artefactos han conservado ambas caras y es casi seguro que se deriven de los contextos funerarios del Horizonte Temprano recientemente saqueados.

\subsection{Restos de fauna y botánicos}

Entre los rellenos disturbados de los pozos de huaqueros en todo 1004 se observaron restos de plantas secas bien preservadas. Entre estos restos tenemos maíz (Zea mays), cáscara de maní (Arachis hypogaea), fragmentos de vainas de pacay (Inga feullei), tubérculos de yuca (Manihot esculenta) y semillas de calabaza (Cucurbita sp.). Dada la ubicación del sitio, estas plantas o sus productos fueron traídos al sitio por la gente.

Restos de fauna estuvieron presentes en todos los sectores, pero particularmente concentrados en el Sector B. Los más abundantes fueron huesos de mamíferos grandes, algunos de camélidos, otros posiblemente de venados, incluyendo especímenes jóvenes, aunque también se observaron aves marinas (Sula variegata y familia Sulidae) y huesos de roedores (Sigmodon sp.). En el extremo norte del Sector B se observó una concentración de huesos de camélidos. En todo el sitio 1004, se registraron huesos de camélidos (presumiblemente llama, Lama glama) en cantidades cuatro veces mayor que la de huesos humanos. También se observaron en todos los sectores, conchas marinas diversas y caracoles terrestres (familia Bulimulidae) de las lomas, aunque muy pocos en el Sector D (Fig. 2e), indicando claramente desplazamientos para su transporte al sitio. Conchas de caracoles de lomas fueron encontradas a menudo en densas concentraciones entre los restos de los pozos de huaqueo.

\subsection{La población enterrada}

En 1004 se registraron en detalle los restos de un total de 14 personas. Estos fueron solo los que no estuvieron muy deteriorados por el sol, porque fueron los adecuados para muestrear con fines de análisis isotópico (Cadwallader 2013). Si bien los entierros estuvieron ubicados en los Sectores A, $\mathrm{B}, \mathrm{C}$ y E, fue evidente que hubo una concentración de ellos alrededor del límite entre los Sectores $\mathrm{B}$ y C (Fig. 1). No es posible hacer reflexiones o apreciaciones precisas sobre la arqueología funeraria de 1004, debido a lo disturbado del sitio, pero sí son posibles algunas observaciones generales. Solo se encontraron dos individuos bien conservados, aunque ambos entierros han sido disturbados por los huaqueros. El cuerpo del adulto fue enterrado en posición fetal, con la parte media superior del cuerpo extendida, con las caderas y rodillas flexionadas, el cuerpo fue enterrado en un hoyo más o menos circular (Sector C). Un niño estuvo enterrado en un hoyo circular pequeño y aunque la parte superior del cuerpo estuvo ausente aparentemente por acción de los huaqueros, la posición del resto del cuerpo sugiere que este niño fue enterrado en posición sentada, con las piernas cruzadas (Sector B, Fig. 2g). Ambas posiciones de entierros fueron comunes durante el período de transición (Isla 2009; DeLeonardis 2012). 
Algunos restos humanos estuvieron íntimamente asociados con fragmentos de textiles llanos o decorados, y a veces con restos de plantas como maní y yuca. Se observaron en un individuo adulto femenino adornos personales de plumas atadas en el cabello.

\section{Osteología y patología}

En la medida de lo posible se hicieron observaciones sobre la edad, el sexo y la patología de cada individuo, aunque el número relativamente pequeño estudiado y el carácter incompleto de los restos óseos limitan las conclusiones que pueden extraerse de cada individuo, y sobre la población en general.

De los 14 individuos estudiados, 12 eran adultos y dos juveniles. Solo la mitad de los adultos pudieron ser identificados por sexo (cuatro mujeres o probables mujeres; dos hombres o probables hombres), debido a la carencia de los respectivos indicadores óseos. No fue posible asignar a cinco de los adultos a un determinado grupo de edad. Una amplia gama de edades se observó en los siete individuos adultos restantes: desde adultos jóvenes hasta adultos maduros (Tabla 1). Para los dos jóvenes, en base a la fusión de los huesos largos, se estimó que corresponden al rango de la infancia temprana (v.g. 1-6 Años) (Scheuer y Black 2000).

En tres individuos cuya dentición era observable, dos adultos más jóvenes mostraron algunas patologías dentales, mientras que un varón mayor tenía tres dientes perdidos ante mortem y abscesos múltiples. Otras patologías observadas en los catorce individuos fueron: cifosis en el mismo hombre mayor que mostró las patologías dentales; osteofitos vertebrales en cuatro individuos, uno con artritis ósea en las vértebras lumbares; y una fractura de costilla curada. Si bien es difícil extrapolar el estado de salud de toda una población a partir de los datos de los restos de estas partes de esqueletos, los datos sugieren que probablemente tuvieron vidas activas, lo cual es indicado por su patología vertebral, con el potencial para vivir hasta una edad avanzada.

Tabla 1. Edades de los restos de adultos de 1004 que fueron estudiados*

\begin{tabular}{lcc}
\hline Grupo de edad & Rango de edad (años) & Número de individuos \\
\hline Adulto joven & $18-25$ & 2 \\
Adulto joven mediano & $26-35$ & 1 \\
Adulto mayor mediano & $36-45$ & 3 \\
Adulto maduro & $46-59$ & 1 \\
Adulto de edad desconocida & - & 5 \\
\hline
\end{tabular}

* Las categorías de edad usadas son las de BARC (Biological Anthropology Research Centre). Los métodos usados para establecer la edad de los restos dependen de los elementos esqueléticos disponibles incluyendo los cambios morfológicos en las pelvis (Lovejoy et al. 1985; Brooks y Suchey 1990; Buckberry y Chamberlain 2002) y el extremo esternal de las costillas (İşcan y Loth 1986) así como la fusión de huesos largos (Scheuer y Black 2000).

Solo dos cráneos incompletos se hallaron en 1004. Mientras que uno estuvo muy fragmentado para determinar la forma de cráneo, el otro presentó modificación artificial circunferencial (de acuerdo a Pomeroy et al. 2010). La deformación craneal ha sido observada como una práctica común en los sitios que datan de finales del Horizonte Temprano y al inicio del Período Intermedio Temprano (Cook 1999).

\section{Fechados radiocarbónicos}

El colágeno óseo de tres individuos de este sitio fue datado por radiocarbono (Cadwallader et al. 2015). La gama de fechados $(2 \sigma)$ obtenidos para los tres individuos se encuentran entre 46 a.C. y 116 d.C., correspondientes a Nasca Inicial, tal como se define por Unkel et al. (2012) en su 
estudio exhaustivo de radiocarbono que une estilos de cerámica a fechas absolutas (Fig. 6). Estas fechas podrían sugerir que 1004 fue utilizado por un tiempo relativamente corto. Sin duda estas fechas son compatibles con la mayoría de los materiales culturales Ocucaje 10 y Nasca 1 que hay en el sitio. La significativa cantidad de materiales culturales de la Fase 9 de Ocucaje sugiere que el sitio fue ocupado antes del tiempo crítico de transición Ocucaje 10 — como se ha señalado en otros sitios Paracas como Cerro Max Uhle en Ocucaje (ver Massey 1991) — y/o que aún hay dudas de detalle para ser resueltos en la seriación cerámica de Paracas/Ocucaje y su variación a través del tiempo y del espacio.

\section{Resultados de isótopos estables}

Es posible aplicar análisis de isótopos estables de carbono y nitrógeno a restos humanos y otros para reconstruir la paleodieta y así conocer sobre las prácticas de subsistencia del pasado. Ellos se basan en el principio de "eres lo que comes" y se ha demostrado que trascienden la cadena alimenticia de manera predecible (para revisiones ver Ambrose 1993; Lee-Thorp 2008). Los isótopos de carbono se usan para distinguir los vegetales usados en la dieta con $\mathrm{C}_{3}$, como papas, quinua, calabaza y frijoles que tienen valores de $\delta^{13} \mathrm{C}$ en la región de $-21 \%$ a $-29 \%$; mientras que las plantas $\mathrm{C}_{4}$, como el maíz y kiwicha , han enriquecido los valores de $\delta^{13} \mathrm{C}$ en la región de $-9 \%$ a $-18 \%$ (DeNiro y Hastorf 1985; Tieszen y Chapman 1992; Cadwallader et al. 2012). Isótopos de nitrógeno, por su parte, se pueden utilizar para evaluar el nivel trófico de un individuo dentro de su cadena de comida local, con valores $\delta^{15} \mathrm{~N}$ que se elevan previsiblemente en cada paso de esa cadena alimentaria (DeNiro y Epstein 1981). Los sistemas marinos tienen elevados valores de isótopos de nitrógeno en comparación con los terrestres y también están enriquecidos en valores $\delta^{13} \mathrm{C}$ (Schoeninger y DeNiro 1984). Por lo tanto, los análisis de la flora y la fauna, componentes de la cadena alimenticia, conjuntamente con los de restos humanos son necesarios para interpretar de manera íntegra los indicadores isotópicos registrados (Cadwallader et al. 2012 sobre esto en la Costa Sur de Perú). Los diversos tejidos humanos registran la dieta en las diferentes etapas de la vida: los dientes que representan la dieta de la infancia (Hillson 2002), los huesos registran la dieta promedio durante por lo menos la última década de la vida (Hedges et al. 2007; Rummel et al. 2007) y el pelo la refiere para los últimos meses de vida (Saitoh et al. 1969).

Se extrajeron muestras de huesos de 14 individuos de 1004; tres de ellos proporcionaron muestras de dientes y otros tres: muestras de cabello. El colágeno fue extraído de los huesos y los dientes (dentina) mediante los métodos Longin (1971) y Richards y Hedges (1999). Se analizó la queratina de las muestras de cabello siguiendo el procedimiento propuesto por O'Connell y Hedges (1999). De dos de los individuos se obtuvieron muestras de cabello cortadas en segmentos de 1 centímetro, para observar los cambios en la dieta en lapsos de tiempo de mes por mes (17 y 10 segmentos, respectivamente). Las muestras de la fauna del sitio — siete camélidos, tres aves y un pequeño roedor - fueron analizadas isotópicamente para obtener una base de referencia para comparar con los restos humanos. Los resultados de todos los análisis se presentan en la Tabla 2 y la Fig. 4.

Tres de las muestras de colágeno de hueso humano no produjeron resultados confiables basados en sus proporciones de C:N (DeNiro 1985). Diez de los diecisiete segmentos de cabello que se analizaron de un mismo individuo (número 67) tampoco produjeron resultados aceptables.

El resto de las muestras proporcionaron un valor medio $\delta^{13} \mathrm{C}$ de colágeno óseo de $-15.7 \pm 1.0 \%$ o y un valor medio $\delta^{15} \mathrm{~N}$ de $9.5 \pm 1.5 \%$ o. La media de los valores de isótopos de carbón de los dientes fue de $-16.0 \pm 0.5 \%$ y de los valores de isótopos de nitrógeno fue de $9.8 \pm 0.9 \%$.

Los valores promedio de las muestras de cabello $\left(\delta^{13} \mathrm{C}=-16.8 \pm 0.8 \%\right.$ y $\delta^{15} \mathrm{~N}=9.6 \pm 0.9 \%$ ) de los tres individuos coinciden con los resultados de las muestras de huesos y dientes, cuando se toman en cuenta las diferencias entre estos tipos de tejido (O'Connell et al. 2001). Los resultados 


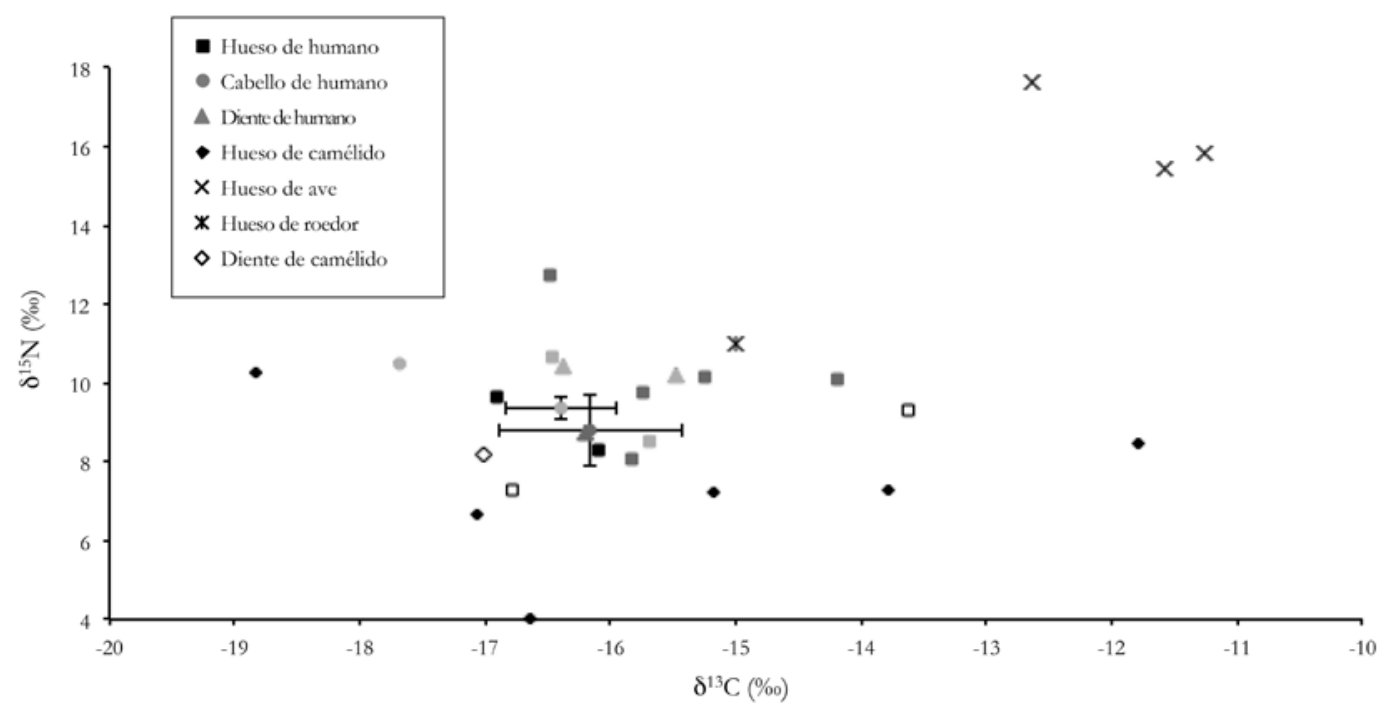

Figura 4. Gráfico ilustrando los resultados del análisis de isótopos de carbón y nitrógeno de 1004. Para los datos de restos humanos el color de los simbolos corresponde a los sectores de donde fue tomada la muestra: negro - Sector A; gris oscuro - Sector $B$; gris claro - Sector C; blanco - Sector E. Los simbolos de las muestras de cabello representan el valor medio por individuo con barras de error que representan una desviación estándar. Todos los restos animales fueron del Sector $B$.

del pelo segmentado dieron promedios individuales de $-16.5 \pm 0.5 \%$ para $\delta^{13} \mathrm{C}$ y $9.2 \pm 0.5 \%$ o para $\delta^{15} \mathrm{~N}\left(\mathrm{n}=12\right.$, Individuo 69) y $-16.2 \pm 0.7 \%$ o para $\delta^{13} \mathrm{C}$ y $8.8 \pm 0.9 \%$ o para $\delta^{15} \mathrm{~N}(\mathrm{n}=7$, Individuo 67$)$. Hubo poca variación $(<2.3 \%$ ) en ambos isótopos a lo largo del cabello de los dos individuos, sugiriendo una dieta relativamente constante en cuanto a isótopos durante el tiempo de crecimiento del cabello: es decir que la dieta no varió mucho con la estación climática (White 1993; Williams y Katzenberg 2012; Webb et al. 2013).

Solo una muestra de colágeno de hueso de camélido no dio resultados aceptables. De los otros seis, el valor medio de $\delta^{13} \mathrm{C}$ fue $-15.6 \pm 2.5 \%$ y el de $\delta^{15} \mathrm{~N}$ fue de $7.3 \pm 2.1 \%$. Los restos de aves arrojaron un valor medio de isótopos de carbono de $-11.8 \pm 0.7 \%$ y un valor medio de isótopos de nitrógeno de $16.3 \pm 1.2 \%$, sustentando su identificación taxonómica como aves marinas. El roedor produjo valores de $-15.0 \%$ y $11.0 \%$ para isótopos de carbono y nitrógeno respectivamente.

\section{Geoglifos}

Se estudiaron cinco geoglifos ubicados sobre la pampa inmediata al oeste del sitio. Estos son pares de líneas rectas que delinean trapezoides en diversas direcciones y una sola línea recta (Fig. 1), con las dimensiones siguientes:

Geoglifo 1: 240 por 10 metros, orientado OSO-ENE;

Geoglifo 2: 350 por 10 metros, más o menos orientado y casi paralelo al Geoglifo 1;

Geoglifo 3: 210 por 10 metros, orientado suroeste-noreste, y cruza el Geoglifo 2. No es posible determinar cuál de los geoglifos fue construido primero (o mantenido más recientemente)

Geoglifo 4: 150 por 20 metros, orientado casi de N-S

Geoglifo 5: Línea de 125 metros de longitud, orientada E-O con el extremo oriental contiguo a un yardang (formación rocosa con efectos de la erosión eólica). 
Tabla 2. Resultados del análisis de isótopos en muestras de humanos y de animales. Los valores indicados en negrita son rechazados debido a una inaceptable proporción C:Ny no se utilizan en las interpretaciones o para calcular los promedios para el cabello

\begin{tabular}{|c|c|c|c|c|c|c|c|c|c|c|c|}
\hline \multirow{2}{*}{$\begin{array}{l}\text { Individuo } \\
\text { (Sector) }\end{array}$} & \multirow[b]{2}{*}{ Sexo } & \multirow[b]{2}{*}{ Edad } & \multicolumn{3}{|c|}{ Hueso } & \multicolumn{3}{|c|}{ Diente } & \multicolumn{3}{|c|}{ Cabello* } \\
\hline & & & $\begin{array}{l}\delta^{13} \mathrm{C} \\
(\% 0)\end{array}$ & $\begin{array}{l}\delta^{15} \mathrm{~N} \\
(\% 0)\end{array}$ & C:N & $\begin{array}{l}\delta^{13} \mathrm{C} \\
(\%)\end{array}$ & $\begin{array}{l}\delta^{15} \mathrm{~N} \\
(\% 0)\end{array}$ & C:N & $\begin{array}{l}\delta^{13} \mathrm{C} \\
(\% o)\end{array}$ & $\begin{array}{l}\delta^{15} \mathbf{N} \\
(\% 0)\end{array}$ & C:N \\
\hline \multicolumn{12}{|l|}{ Humanos } \\
\hline $6(B)$ & $\mathrm{U}$ & YA & $-16.5^{1}$ & 12.8 & 3.3 & & & & & & \\
\hline $60(\mathrm{~A})$ & F? & Adulto & $-17.6^{2}$ & 9.4 & 4.5 & & & & & & \\
\hline $61(\mathrm{~A})$ & $\mathrm{U}$ & Adulto & $-16.1^{3}$ & 8.3 & 3.5 & & & & & & \\
\hline $62(\mathrm{~A})$ & $\mathrm{U}$ & Adulto & $-16.9^{4}$ & 9.6 & 3.6 & & & & & & \\
\hline 63 (B) & U & OMA & $-14.2^{5}$ & 10.1 & 3.4 & & & & & & \\
\hline 64 (B) & $\mathrm{U}$ & Adulto & $-15.3^{6}$ & 10.2 & 3.2 & & & & & & \\
\hline 65 (B) & J & $\mathrm{EC}$ & $-15.7^{6}$ & 9.8 & 3.4 & & & & & & \\
\hline 66 (B) & $\mathrm{F}$ & YMA & $-15.8^{4}$ & 8.1 & 3.4 & $-16.2^{a}$ & 8.7 & 3.4 & & & \\
\hline \multirow[t]{18}{*}{67 (B) } & $\mathrm{F}$ & MA & $-16.1^{2}$ & 9.4 & 3.9 & & & & -15.2 & 9.2 & 4.0 \\
\hline & & & & & & & & -15.5 & 9.2 & 4.0 & \\
\hline & & & & & & & & -15.7 & 9.1 & 3.9 & \\
\hline & & & & & & & & -15.9 & 9.1 & 3.9 & \\
\hline & & & & & & & & -15.7 & 8.8 & 3.8 & \\
\hline & & & & & & & & -15.8 & 7.8 & 3.8 & \\
\hline & & & & & & & & -15.6 & 7.5 & 3.8 & \\
\hline & & & & & & & & -15.4 & 8.5 & 3.8 & \\
\hline & & & & & & & & -16.5 & 9.5 & 3.8 & \\
\hline & & & & & & & & -17.3 & 9.7 & 3.8 & \\
\hline & & & & & & & & -16.9 & 9.7 & 3.8 & \\
\hline & & & & & & & & -16.4 & 9.6 & 3.9 & \\
\hline & & & & & & & & -16.0 & 9.4 & 3.9 & \\
\hline & & & & & & & & -15.8 & 9.1 & 3.9 & \\
\hline & & & & & & & & -16.6 & 8.8 & 3.9 & \\
\hline & & & & & & & & -16.4 & 8.8 & 3.9 & \\
\hline & & & & & & & & -17.1 & 8.8 & 3.9 & \\
\hline & & & & & & & $=$ & $\begin{array}{l}-16.2 \\
\pm 0.7\end{array}$ & $\begin{array}{l}8.8 \\
\pm 0.9\end{array}$ & $(\mathrm{n}=7)$ & \\
\hline $68(C)$ & M & OMA & $-16.5^{7}$ & 10.7 & 3.4 & $-16.4^{a}$ & 10.4 & 3.2 & -17.7 & 10.5 & 3.7 \\
\hline
\end{tabular}


Tabla 2. Resultados del análisis de isótopos en muestras de humanos y de animales. Los valores indicados en negrita son rechazados debido a una inaceptable proporción C:Ny no se utilizan en las interpretaciones o para calcular los promedios para el cabello

\begin{tabular}{|c|c|c|c|c|c|c|c|c|c|c|c|}
\hline & & & & Hueso & & & Diente & & & Cabello* & \\
\hline $\begin{array}{l}\text { Individuo } \\
\text { (Sector) }\end{array}$ & Sexo & Edad & $\begin{array}{l}\delta^{13} \mathrm{C} \\
(\% 0)\end{array}$ & $\begin{array}{l}\delta^{15} \mathrm{~N} \\
(\% 0)\end{array}$ & C:N & $\begin{array}{l}\delta^{13} \mathrm{C} \\
(\% \circ)\end{array}$ & $\begin{array}{l}\delta^{15} \mathrm{~N} \\
(\% 0)\end{array}$ & C:N & $\begin{array}{l}\delta^{13} \mathrm{C} \\
(\% 0)\end{array}$ & $\begin{array}{l}\delta^{15} \mathrm{~N} \\
(\% \mathrm{o})\end{array}$ & C:N \\
\hline 69 (C) & M? & YA & $-15.7^{6}$ & 8.5 & 3.3 & $-15.5^{b}$ & 10.2 & 3.2 & -17.5 & 8.6 & 3.7 \\
\hline & & & & & & & & -17.2 & 9.3 & 3.7 & \\
\hline & & & & & & & & -16.7 & 9.5 & 3.7 & \\
\hline & & & & & & & & -16.5 & 9.7 & 3.7 & \\
\hline & & & & & & & & -16.5 & 9.5 & 3.7 & \\
\hline & & & & & & & & -16.1 & 9.6 & 3.7 & \\
\hline & & & & & & & & -16.1 & 9.3 & 3.7 & \\
\hline & & & & & & & & -15.9 & 9.0 & 3.7 & \\
\hline & & & & & & & & -16.1 & 8.9 & 3.7 & \\
\hline & & & & & & & & -16.4 & 8.1 & 3.6 & \\
\hline & & & & & & & $=$ & $\begin{array}{l}-16.4 \\
\pm 0.4\end{array}$ & $\begin{array}{l}9.4 \\
\pm 0.3\end{array}$ & $(\mathrm{n}=8)$ & \\
\hline 70 (E) & $\mathrm{F}$ & OMA & $-16.8^{4}$ & 7.3 & 3.5 & & & & & & \\
\hline 71 (E) & $\mathrm{J}$ & EC & $-13.6^{8}$ & 9.3 & 3.2 & & & & & & \\
\hline $72(\mathrm{E})$ & $\mathrm{U}$ & Adulto & $-16.6^{8}$ & 10.3 & 3.8 & & & & & & \\
\hline Fauna & & & & & & & & & & & \\
\hline AN4 & & & -13.8 & 7.3 & 3.3 & & & & & & \\
\hline AN5 & & & -18.8 & 10.3 & 3.3 & & & & & & \\
\hline AN6 & & & -11.8 & 8.4 & 3.3 & & & & & & \\
\hline AN7 & & & -16.6 & 4.0 & 3.3 & & & & & & \\
\hline AN8 & & & -17.1 & 6.6 & 3.6 & & & & & & \\
\hline AN9 & & & -15.2 & 7.3 & 3.5 & & & & & & \\
\hline AN11 & & & -12.6 & 17.6 & 3.4 & & & & & & \\
\hline AN12 & & & -11.3 & 15.8 & 3.2 & & & & & & \\
\hline AN13 & & & -15.0 & 11.0 & 3.4 & & & & & & \\
\hline AN14 & & & -14.5 & 20.9 & 4.2 & & & & & & \\
\hline AN15 & & & -11.6 & 15.4 & 3.2 & & & & & & \\
\hline AN16 & & & & & & -17.0 & 8.2 & 3.2 & & & \\
\hline
\end{tabular}

${ }^{1}$ Costilla; ${ }^{2}$ Fémur; ${ }^{3}$ Peroné; ${ }^{4}$ Tibia; ${ }^{5}$ Cúbito; ${ }^{6}$ Húmero; ${ }^{7}$ Cráneo; ${ }^{8}$ Vértebra ${ }^{\mathrm{a}} \mathrm{M} 1 ;{ }^{\mathrm{b}} \mathrm{M} 3$

*Valores de muestras de cabello segmentado desde lo proximal a lo distal 
Cada geoglifo está formado por líneas rectas constituidas por pequeñas piedras acumuladas, las que, aunque no se encuentran en la abundancia que presenta la superficie de los sitios con geoglifos de la cuenca del río Grande, están fácilmente disponibles en la superficie de la pampa (Figs. 5a y 5c). Al parecer, la superficie entre los pares de líneas fue limpiada de las piedras más grandes, práctica observada en geoglifos de otras partes de la Costa Sur, por ejemplo las atribuidas al período de transición Paracas-Nasca determinado por la cerámica asociada o varios métodos de fechado (v.g. Silverman 1990; Greilich y Wagner 2009; Stanish et al. 2014). Si se «limpia» la superficie de esta pampa desértica de la delgada cubierta de materiales acarreados por el viento, queda expuesto el color amarillo brillante de la superficie subyacente, resultando tan llamativo que especulamos si pudo haber sido parte de la concepción original del diseño de estos geoglifos. La pampa en la que se encuentran estos geoglifos, también presenta restos de varias estructuras semicirculares pequeñas, construidas con piedras de formas irregulares (Figs. 1 y 5b); las que en otras partes de la Costa Sur están típicamente asociadas con los geoglifos, y son comúnmente interpretadas como "paravientos» para ofrecer refugio temporal en este entorno extremadamente ventoso (Clarkson 1990).

\section{Discusión}

\section{¿Qué tipo de sitio fue 1004?}

Como se ha señalado anteriormente, el registro arqueológico de Paracas Transicional en 1004 incluye muchos elementos utilitarios como piedras de moler (batanes), artefactos líticos, redes de pesca, implementos agrícolas de madera y recipientes para cocinar. También incluye algunos materiales de construcción tales como postes de huarango y esteras de caña, junto con abundantes evidencias de alimentos vegetales desecados, moluscos marinos y terrestres, así como huesos de animales. De hecho, el sitio está casi definido por su abundancia de huesos de camélidos. En conjunto, estos podrían sugerir el tipo de cultura material y basural que permanece asociado a algún tipo de sitio habitacional. En relación a ello, Cook (1999) sugiere que la presencia de quincha se puede utilizar para indicar la arquitectura residencial en los sitios de este período.

1004 también incluye varios entierros humanos y considerando que comúnmente se enterró a la gente con los artefactos y otros que podrían necesitar para la vida después de la muerte, la presencia de estos materiales no excluye la posibilidad de que solo sea un cementerio saqueado (con o sin otros fines rituales asociados). La variedad de artefactos que reportamos en 1004 es similar a los relatados por Silverman (2002b) de la excavación de Rubini, de un cementerio de la Fase Ocucaje 10 en la cuenca de Ocucaje. Cook (1999), en su estudio del valle de Ica seńala que los sitios suelen incluir testimonios arquitectónicos tanto de actividades ceremoniales como habitacionales que se realizan dentro de un mismo sitio.

En resumen, si bien no podemos estar seguros de qué tipo de sitio fue 1004, porque no lo hemos excavado y ha sido saqueado intensamente, nuestra interpretación tentativa es que sí presenta un componente habitacional permanente o semipermanente, en razón de: 1) los aparentemente pocos entierros humanos en relación con el tamaño total del sitio; 2) la presencia de grandes batanes; 3) la cantidad de huesos de camélidos cuatro veces mayor que la de humanos en nuestras colecciones; y a 4) las grandes cantidades de restos de la vida cotidiana en el sitio.

\section{¿Por qué 1004 se ubicó en ese lugar?}

Si las evidencias de 1004 son realmente de ocupación permanente o temporal, entonces su ubicación en lo alto, por encima de la planicie aluvial del río, en las márgenes de la Cuenca de Samaca, es semejante a otros sitios Paracas conocidos en el valle de Ica. Menzel et al. (1964: 104) describen cómo los sitios con cerámica de la fase Ocucaje 8 se colocaron a lo largo de las laderas escarpadas del valle, incluyendo un sitio fortificado que se extiende por varios kilómetros de largo y sobre un 

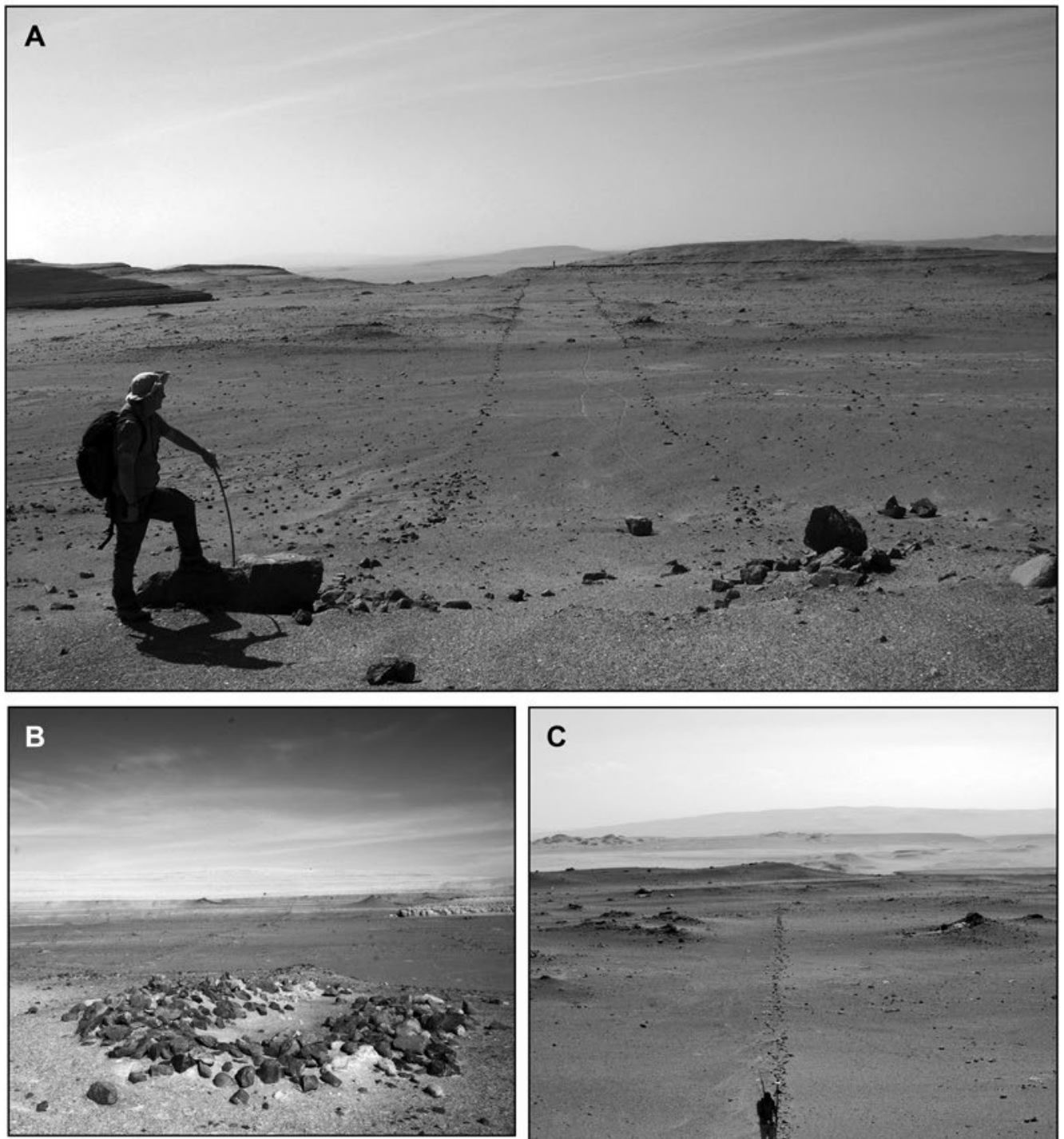

De arriba a abajo:

A Vista al noreste de geoglifo 1.

B Paraviento en el extremo noreste del geoglifo 2.

C Vista al este del geoglifo 5.

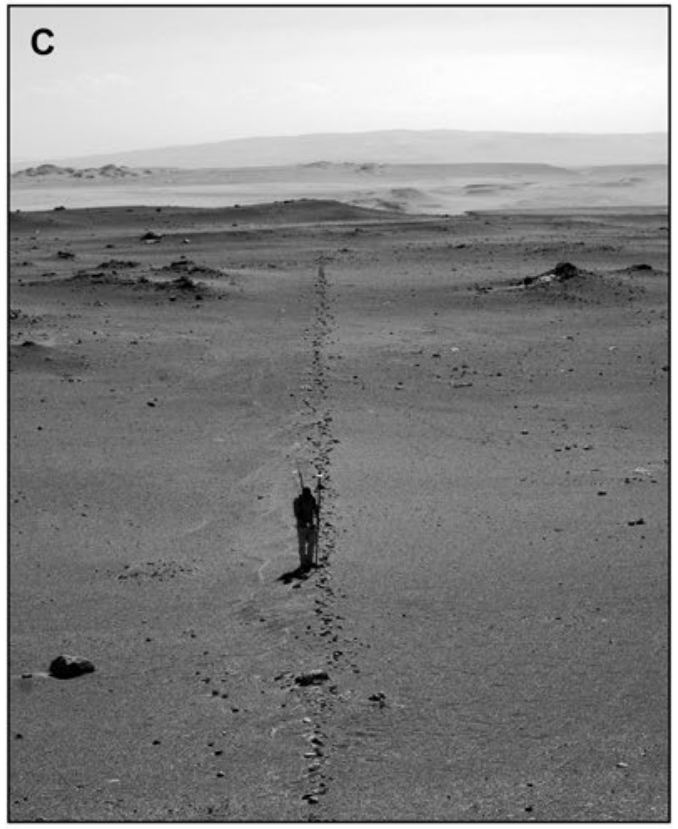

Figura 5. Ejemplos de geoglifos y de paravientos asociadas sobre la pampa ubicada justo arriba de 1004 (Fotografias de D. Beresford-Jones). 


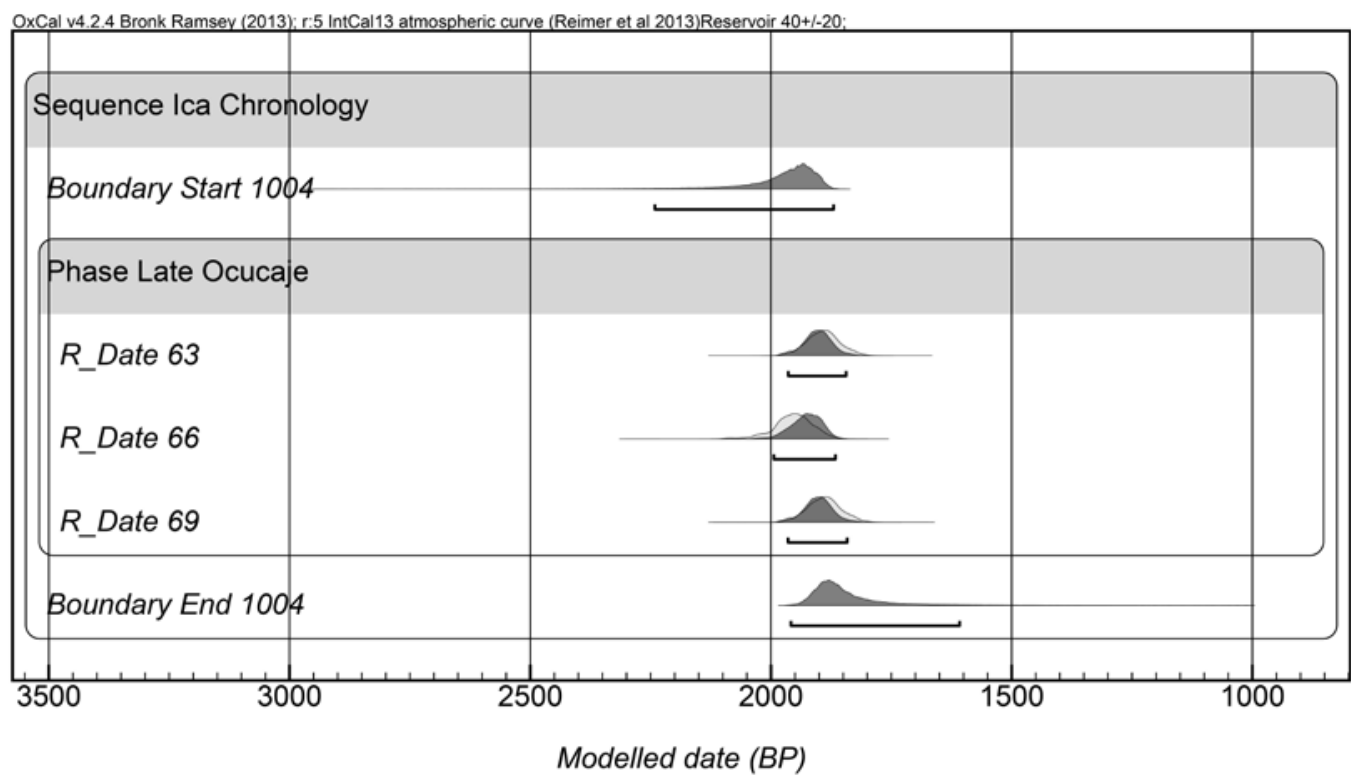

Figura 6. Fechados de radiocarbono para el sitio de 1004. Calibrado mediante la curva IntCal13 (Reimer et al. 2013), con corrección (Hogg et al. 2009) en OxCal versión 4.2 (Bronk Ramsey 2009). Todas las muestras son de colágeno de óseo bumano.

gran cerro cerca de Santa Lucía. Este sitio fue ocupado hasta el siguiente período durante las fases de Nasca Temprano (3 y 4), aunque con el tiempo, en las fases posteriores, se construyó en las partes más bajas de las laderas (Massey 1991). Sitios fortificados de la época de transición Ocucaje 9/10 también se describen en los cerros, por encima de La Venta, Santa Lucía y más notablemente en el gran sitio fortificado de Tajahuana en Ocucaje, el presunto centro de poder de la época Paracas (Menzel et al. 1964; Williams 1980). Sin embargo asentamientos más pequeños también ocupan las laderas bajas de los cerros (Menzel et al. 1964, Massey 1991, Cook 1999). Mientras que en Nazca para la fase Nasca 1 Transicional, se registraron asentamientos en las laderas del Cerro Carapo en Palpa y en el sitio 220 en la Cuenca de Ingenio (Silverman 2002a: 164).

La práctica de ubicar los sitios en lo alto, por encima del piso del valle, habría tenido su origen mucho antes, en el Horizonte Temprano. Wallace (1962) señala que, a semejanza de otros sitios que había observado, el de Cerrillos, de las Fases 3 y 4 de Ocucaje, en el valle medio, está inconvenientemente situado sobre una empinada ladera. Wallace no seńala evidencia de fortificaciones o estructuras defensivas en Cerrillos y Menzel et al. (1964: 5) sugieren que la razón de su ubicación podría ser la de conservar los terrenos del fondo del valle para la producción agrícola. Del mismo modo, Cook (1999) señala que los sitios Paracas Medio (fases 5 a 8) en Ocucaje, están ubicados en la cima de los cerros bajos y alejados, que bordean el río Ica, sin indicios de ubicaciones en el piso del valle, interpretándolos como lugares utilizados para actividades públicas sobre la base de su arquitectura de adobe y la proximidad de los geoglifos, sin presentar evidencias de ninguna estructura defensiva.

La explicación más mesurada de la ubicación de los sitios Paracas en lo alto del valle bajo de Ica, por encima de las llanuras aluviales del río, es que fue para su defensa, ya que la fortificación y ubicaciones defendibles son una característica común de muchos de esos sitios (Williams 1980). No hay evidencia de fortificaciones en 1004. Sin embargo, el sitio cuenta con líneas de visión de largas distancias, tanto hacia arriba como hacia el fondo del valle, lo que permitiría inferir, por tanto, que era de mayor importancia para sus habitantes que la proximidad a los recursos esenciales, 
incluyendo el agua. De hecho, esas interpretaciones concuerdan con nuestra comprensión de la época como una de convulsión social, evidente en otros aspectos del registro arqueológico de las cuencas de Callango y Ocucaje. En base a datos del patrón de asentamiento, se planteó la hipótesis de la existencia de una confederación de unidades sociopolíticas en todo el valle durante la época Paracas (Massey 1991; DeLeonardis 2005). Massey (1991) infiere cambios en el asentamiento y por lo tanto de poder entre los principales sitios de Callango, pasando de Ánimas Bajas en la Fase 8 de Ocucaje, a Ánimas Altas para la Fase 9, sitio último que habría sido abandonado ritualmente, pasando el poder a Cerro Max Uhle en la cuenca de Ocucaje. Menzel et al. (1964) conciben a Ocucaje como el centro del poder durante la Fase 10 de Ocucaje, argumentando que los cambios concurrentes en los estilos de cerámica y la disminución de su diversidad reflejan las modificaciones en las relaciones políticas. Durante este período crítico de transición, muchos de los sitios más grandes y extensos en el valle bajo, al parecer fueron abandonados y la población del valle de Ica se dispersó en pequeños sitios a lo largo del valle (Massey 1991). En efecto, Massey (1991) considera que la cuenca de Callango va quedando abandonada en gran parte con el colapso y la descentralización de la autoridad regional en ese momento. Mientras tanto, la cuenca de Ocucaje aparentemente no fue afectada, con el sitio de Cerro Max Uhle continuamente ocupado, sin evidencia clara de cambios de la población, pero con nuevos sitios establecidos para hacer frente a una expansión de la población (Massey 1991).

La riqueza de hallazgos de posibles armas de este período también podría sugerir evidencias de un ambiente social hostil, lo que se infiere por ejemplo, a partir de la forma y la deposición de puntas de obsidiana en los pisos de Ánimas Altas, poco antes de su abandono (Massey 1991; Burger 2007), así como en los cambios en la ubicación de los sitios (Cook 1999). La abundancia de puntas de proyectil de obsidiana en 1004 también podría interpretarse como evidencia de actividades agresivas. Se considera que la hostilidad y violencia percibidas en Paracas Transicional continúan ampliamente en el período Nasca con la tradición de las cabezas-trofeo, evidente tanto en su iconografía como en el más amplio registro arqueológico (DeLeonardis y Lau 2004).

Sin embargo, estas interpretaciones deben ser tomadas con cierta cautela. Menzel et al. (1964: 177) concluyeron que el estilo, la iconografía y las técnicas de producción artesanal eran tan uniformes a través del valle de Ica durante de la fase Ocucaje 9, que permitía sugerir un período de unificación social. Por su parte, Bachir Bacha y Llanos (2013: 200-201) proponen que las elites de Ánimas Altas tenían la capacidad de llamar y reunir diversos grupos sociales de lugares alejados para celebrar eventos religiosos, públicos y ritos familiares. Ellos citan una heterogeneidad estilística y técnica en la muestra cerámica y las diferencias en formas de deformación craneal presentes en el sitio como evidencia de la presencia de diversos grupos sociales en Ánimas Altas, lo cual además les lleva a proponer al sitio como centro de peregrinación Paracas. Por otra parte, no podemos tener la certeza de que las «armas» de obsidiana no fueron usadas principalmente o aun exclusivamente para la caza de animales (un análisis adicional sobre el tipo de sangre detectado en una punta de proyectil de 1004 puede ser esclarecedor al respecto). Silverman (1996) en su revisión sobre el Período Formativo en la Costa Sur, describe sitios de la Fase 8-10 de Ocucaje en el valle bajo de Ica, ubicados principalmente en la llanura aluvial del valle, cerca de las fuentes de agua que sustentan la vida en este desierto.

Realmente, hay otros factores a tomar en cuenta para comprender la ubicación de 1004, los mismos que no necesariamente descartan la idea de que esa ubicación fue elegida por sus impresionantes vistas, pero que sin embargo mitigan la impresión de lo inhóspito que hoy parece. Por ejemplo, la vegetación de las cuencas del Valle bajo de Ica ha sufrido un cambio considerable en los últimos dos milenios (Beresford-Jones et al. 2011). Sostenemos que estos cambios implicaron alteraciones significativas en la morfología de la llanura aluvial del río, como consecuencia de su exposición a eventos de inundación de alta energía y subsiguientes épocas de sequía hacia el final del Período Intermedio Temprano. Existe abundante evidencia de que anteriormente el río Ica pueda 
haber ocupado un curso diferente, más alto, y que bosques riparios, mucho más densos, puedan haberse extendido mucho más arriba en los flancos de estas cuencas, sobre todo en forma de troncos de árboles relictos, los que reflejan con claridad capas freáticas a mayor altura en el pasado (Cook 1999; Beresford-Jones 2011). La ubicación de 1004, por lo tanto, puede haber estado mucho más cerca de la vegetación del valle de lo que está ahora. Además, los huarangos (Prosopis limensis), especies arbóreas clave de este entorno, crecen a un tamaño enorme en forma de bosque primario imperturbado, y pueden extenderse considerablemente hasta los flancos de estas cuencas riparias. En conjunto, estos árboles tienen importantes implicancias con respecto a cuán lejos se encontraba originalmente 1004 de recursos vitales como sombra, agua y comida.

\section{¿Que nos dice 1004 sobre la vida diaria durante la época Paracas?}

El registro arqueológico de 1004, como sitio Paracas, presenta muchas evidencias de la vida cotidiana, incluyendo batanes, redes de pescar y vasijas usadas para cocinar. Estos artefactos y sus restos asociados botánicos y de fauna, muestran que los habitantes de 1004 (o los individuos enterrados allí) recolectaron recursos silvestres del mar, bosque seco y lomas, y cultivaron plantas y animales domesticados, los que jugaron un rol en su subsistencia. Por lo tanto, parece que su población practicó una economía broad spectrum de caza, recolecta, pesca y agricultura, y es imposible evaluar la importancia relativa de cada actividad sin mayores datos.

Sin embargo, los análisis de isótopos arrojan alguna luz en este sentido. Los valores de isótopos de carbón y nitrógeno en el colágeno de los individuos enterrados en 1004 indican una dieta predominantemente terrestre, con recursos marinos jugando un papel insignificante, a pesar de la notoria presencia de conchas marinas en este sitio y otros en el valle de Ica (DeLeonardis y Glascock 2013). Los resultados de isótopos de cabello, por un lado, y de la combinación de los resultados de los diferentes tipos de tejidos, no sugieren una variación estacional importante en la alimentación, ni cambios alimenticios durante el período de vida de los individuos de 1004 (aunque esta falta de variación estacional se observó en muestras de solo dos individuos que representan 7 y 10 meses respectivamente, de modo que es posible que este resultado sea por el tamaño de la muestra). Si estas firmas isotópicas son una representación confiable de la obtención de alimentos de Paracas Transicional, entonces, al igual que las evidencias de fauna, vegetales y artefactos, ellas confirman que los pobladores estuvieron explotando una variedad de recursos isotópicamente diversos, pero también que estuvieron almacenando componentes significativos de sus alimentos y así mantuvieron una continuidad isotópica en su dieta. De hecho, esto mismo también sugiere que la gente que está enterrada en 1004, residió permanentemente en algún lugar, a donde fueron llevados los diferentes recursos de varias zonas (como del mar, las lomas, y la llanura inundable ribereña), por supuesto moviéndose entre los sitios situados en aquellas zonas.

Hay mucha variación en los valores de isótopos de los camélidos analizados. Un espécimen (AN5) tiene un valor inusualmente alto $\delta^{15} \mathrm{~N}$ (10.3\%o): 4\%o superior al valor $\delta^{15} \mathrm{~N}$ medido en plantas de ecologías de lomas (Thornton et al. 2011). Un incremento de 4\%o en Nitrógeno es la diferencia entre dos niveles en la cadena alimentación (DeNiro y Esptein 1981) y esto sugiere que este individuo puede haber consumido principalmente plantas de lomas. Al ser el único en la muestra de camélidos, sugerimos podría ser de guanaco silvestre cazado. Los especímenes restantes de camélidos son, por lo tanto, interpretados como restos de especies domesticadas — probablemente de llama-. Sin embargo, la amplia gama en $\delta^{13} \mathrm{C}$ y $\delta^{15} \mathrm{~N}(-11.8 \%$ a $-17.1 \%$ y $4.0 \%$ a $8.4 \%$ respectivamente) en estos animales sugiere que ellos no eran alimentados con forraje en un lugar restringido. Por lo tanto, el manejo activo del rebaño para alimentarlo con forraje probablemente fue mínimo. Se han identificado restos de llama y guanaco en sitios de Paracas y de Nasca Temprano antes (Renneberg et al. 2009) y son evidentes en la iconografía de la época, pero poco se sabe sobre el manejo de animales durante este tiempo transicional. 
Las especies de aves identificadas, Sula variegata o el piquero peruano, es un ave exclusivamente marina (BirdLife International 2014), como lo han sugerido sus valores isotópicos. Su presencia añade a la interpretación de que los recursos de la costa fueron explotados en una variedad de recursos alimenticios.

En suma, los datos de 1004 sugieren que la caza y la recolección siguieron siendo importantes para estos agricultores y pastores, lo que coincide con lo observado en otros sitios de períodos similares (Wallace 1962; Lumbreras 1974: 59; DeLeonardis 2005; Beresford-Jones 2011; Cadwallader et al. 2012).

\section{¿Qué nos dicen los geoglifos sobre 1004?}

Los geoglifos son una característica común de las pampas del desierto existente entre los valles fluviales de la Costa Sur. Los cinco ubicados en la zona inmediata a 1004 solo son parte de un palimpsesto más grande con marcas antiguas que pueden rastrearse a lo largo de ambas laderas del valle bajo de Ica. Por supuesto, no tenemos indicadores específicos para las fechas de estos geoglifos, pero por su cercana asociación espacial resulta posible que hubieran sido construidos en alguna asociación con 1004 .

El papel de los geoglifos ha sido un tema de gran especulación e interés por arqueólogos y otros. Silverman (1990) interpreta los geoglifos de la región de Nazca como transformantes de un paisaje natural abierto, a un paisaje humano cerrado. Esto integró el paisaje a las actividades humanas vinculadas a la religión. Silverman sugiere que la construcción de los geoglifos habría sido a través del trabajo comunal de la mit’a, lo cual implicaba la contribución de cada ayllu o grupo familiar a la construcción de glifos individuales. La construcción y mantenimiento de los glifos, por consiguiente, era un acontecimiento social, político y religioso a la vez, como el sistema de ceques utilizado por los Incas, que ha visto grupos de parentesco manteniendo partes específicas de las líneas que se irradian desde el Cuzco, uniendo partes de un paisaje sagrado (Aveni 1990). Por lo tanto, con el tiempo, los diferentes grupos construyendo geoglifos diversos darían lugar al palimpsesto aparentemente desordenado y hoy visible en las superficies de la pampa. Muchos de los geoglifos se dirigen a, o finalizan en, cerros $\mathrm{u}$ otras prominencias que pueden ser interpretados como rasgos del paisaje sagrado o huacas. Reinhard (1993) señala que ciertos grupos andinos aún siguen líneas orientadas hacia los cerros en procesiones acompañadas por músicos como parte de rituales para asegurar la fertilidad agrícola.

Stanish y sus colegas recién han descrito geoglifos en el Valle de Chincha, muchos de los cuales parecen idénticos a los geoglifos trapezoidales cercanos a 1004 (Stanish et al. 2014, Stanish y Tantaleán 2018). Sobre la base de su análisis de una diversidad de formas de geoglifos en Chincha, hacen nuevas propuestas sobre sus funciones, que incluyen algunos alineados con movimientos solares y asociados con sitios rituales Paracas, y otros que actúan como guías para trasladarse a través de la pampa del desierto.

Las similitudes en forma y ubicación de los geoglifos trapezoidales cercanos a 1004 con los reportados en Chincha, son llamativos. Los de Samaca corren en varias direcciones y no están claramente alineados con los enormes yardangs (formaciones rocosas con efectos de la erosión eólica) que son los que caracterizan y definen el paisaje del tablazo de Ica. De ser así, esto permite una interpretación alternativa sobre la naturaleza del sitio 1004: que más que un sitio de habitación permanente, fue un sitio de alguna forma de ocupación temporal al servicio de fines rituales (incluyendo por supuesto entierros) y/o actividades relacionadas a la preparación de rituales llevados a cabo en el área con geoglifos. Ante tal escenario, artefactos hallados en 1004 como batanes y huesos de camélidos podrían considerarse como para ofrendas u otros eventos rituales que tuvieron lugar ahí. Mientras tanto, tendríamos que buscar en otra parte para encontrar su sitio de ocupación permanente, sugerido por los datos isotópicos. 
Naturalmente todas estas interpretaciones así como la asociación adoptada entre 1004 y los geoglifos requieren de mayores investigaciones para ser resueltas. Sin embargo, seguramente los autores de los geoglifos deben haber estado advertidos de la existencia del sitio 1004. En realidad, no tenemos que asumir contemporaneidad entre los geoglifos y el sitio para asumir una relación entre los dos; considerando por ejemplo si los geoglifos habrían sido elaborados mucho después de Paracas como parte de una forma de culto ancestral para los entierros de 1004.

\section{Conclusiones}

A pesar de las limitaciones de nuestro estudio y de la gran incertidumbre para precisar el tipo de ocupación que representa 1004 y de su condición de saqueado, hay varias líneas de datos que nos pueden ofrecer una visión de la vida cotidiana de las poblaciones de la cuenca de Samaca actualmente.

La estrategias de subsistencia de sus habitantes (o de los individuos enterrados en el sitio) fueron variadas y aparentemente hicieron uso de todos los recursos disponibles mediante la caza y la recolecta de recursos marinos y de lomas, así como la agricultura y el pastoreo en oasis riparios: algunos de los que a la fecha no han sido demostrados o evidenciados muy claramente en otros sitios del mismo período. Por lo visto, a diferencia de las poblaciones contemporáneas río arriba o de las poblaciones nasca que siguieron, las poblaciones paracas transicional en la cuenca de Samaca, según parece, estuvieron practicando la agricultura además de contar con un amplio espectro de otros recursos disponibles. Por lo tanto, ellos debieron haber sido regularmente móviles y esto también podría ser sugerido relativamente por la cantidad de obsidiana presumiblemente desde las distantes fuentes de Quispisisa. La limitada evidencia de isótopos en la dieta de 1004 no indica estrategias de subsistencia particularmente dictadas por la estacionalidad, sugiriendo en cambio un manejo importante del medioambiente y almacenaje de productos alimenticios esenciales.

En muchos otros aspectos — su ubicación, ciertas evidencias de las prácticas de entierro y la presencia de puntas de obsidiana- 1004 parece ser similar a otros sitios de este período en la Costa Sur. Por lo tanto, con seguridad, las poblaciones de la cuenca de Samaca estuvieron participando de, e influenciadas por, los más grandes sucesos socioculturales y políticos que tuvieron lugar en la Costa Sur del Perú como el fin del Horizonte Temprano y el inicio a la transición a Nasca, en el período Intermedio Temprano. Además, al mismo tiempo, los ritmos cotidianos de sus vidas siguieron reflejando la explotación antigua y exitosa de los diversos medioambientes entre los que ellos vivieron.

\section{Agradecimientos}

Esta investigación fue financiada por una beca otorgada a Lauren Cadwallader por el Consejo de Investigación de Artes y Humanidades. Agradecemos a Alberto Benavides y la gente de Samaca por su asistencia durante el trabajo de campo. También agradecemos al Ministerio de Cultura del Perú por otorgar el permiso para el trabajo de campo y los subsiguientes análisis de las muestras. Gracias también a nuestras colegas Tamsin O'Connell y Catherine Kneale de la Universidad de Cambridge, Fraser Sturt de la Universidad de Southampton y George Chauca Iparraguirre por sus contribuciones importantes a este trabajo. Asimismo, agradecemos a la NERC-AHRC National Radiocarbon Facility (NRCF), UK, por la financiación de la datación por radiocarbono

\section{Nota}

1 Este análisis fue realizado por cortesía de la Dra. Gladys Ocharán en el Laboratorio de Microscopía Electrónica y Aplicaciones en el Perú (MYAP, www.myapcompany.com) 


\section{REFERENCIAS}

\section{Ambrose, S. H.}

1993 Isotopic analysis of paleodiets: Methodological and interpretive considerations, en: M. K. Sandford (ed.), Investigations of ancient human tissue: Chemical analyses in anthropology, 59-130, Gordon and Breach, Philadelphia.

Aveni, A. F.

1990 Order in the Nazca lines, en: A. F. Aveni (ed.), The lines of Nazca, 41-114, The American Philosophical Society, Philadelphia.

\section{Bachir Bacha, A.}

2017 El edificio de los frisos de animas altas. Ser paracas en el valle bajo de Ica, Boletin de Arqueología PUCP 22, 191-225. https://doi.org/10.18800/boletindearqueologiapucp.201701.008

Bachir Bacha, A. y O. D. Llanos

2013 ¿Hacia un urbanismo Paracas en Ánimas Altas/Ánimas Bajas (Valle de Ica)?, Boletín de Arqueología PUCP $17,169-204$.

Beresford-Jones, D.G.

2011 The lost woodlands of ancient Nasca. A case-study in ecological and cultural collapse, Oxford University Press, Oxford. https://doi.org/10.5871/bacad/9780197264768.001.0001

Beresford-Jones, D. G., O. Q. Whaley, C. Alarcón y L. Cadwallader, L

2011 Two millennia of changes in human ecology: Archaeobotanical and invertebrate records from the lower Ica Valley, south coast Peru, Vegetation History and Archaeobotany 20, 273-292. https://doi.org/10.1007/ s00334-011-0292-4

\section{Birdlife International}

2014 Species factsheet: Sula variegate, descargado de http://www.birdlife.org el 03/04/2014

\section{Bronk Ramsey C.}

2009 Bayesian analysis of radiocarbon dates, Radiocarbon 51, 337-360. https://doi.org/10.1017/ S0033822200033865

Brooks, S. y J. M. Suchey

1990 Skeletal age determination based on the os pubis: a comparison of the Acsádi-Nemeskéri and Such Brooks methods, Human Evolution 5, 227-238. https://doi.org/10.1007/BF02437238

Buckberry, J. L. y A. T. Chamberlain

2002 Age estimation from the auricular surface of the ilium: a revised method, American Journal of Physical Anthropology 119, 231-239. https://doi.org/10.1002/ajpa.10130

Burger, R. L.

2007 Late Paracas obsidian tools from Animas Altas, Peru, Andean Past 8, 477-492.

Burger, R. L. y M. D. Glascock

2000 Locating the Quispisisa obsidian source in the department of Ayacucho, Latin American Antiquity 11, 258-268. https://doi.org/10.2307/972177

\section{Cadwallader, L.}

2013 Investigating 1500 years of dietary change in the lower Ica Valley, Peru, using an isotopic approach, tesis de doctorado, Departamento de Arqueología, Universidad de Cambridge.

Cadwallader, L., D. G. Beresford-Jones, O. Q. Whaley y T. C. O'Connell

2012 The signs of maize? A reconsideration of what $\delta^{13} \mathrm{C}$ values say about palaeodiet in the Andean region, Human Ecology 40, 487-509. https://doi.org/10.1007/s10745-012-9509-0

\section{Cadwallader, L., S. Arce T., T. C. O’Connell, A. G. Pullen y D. G. Beresford-Jones}

2015 Dating the dead: New radiocarbon dates from the Lower Ica Valley, south coast Peru, Radiocarbon 57, 765-773. https://doi.org/10.2458/azu_rc.57.18343 
Cadwallader, L., D. G. Beresford-Jones, F. C. Sturt, A. G. Pullen y S. Arce T.

2018 Doubts about how the Middle Horizon collapsed (c. AD 1000) and Other insights from the looted cemeteries of the lower Ica Valley on the South Coast of Peru, Journal of Field Archaeology 43, 316-331. https://doi.org/10.1080/00934690.2018.1464306

Canziani, J.

2013 Arquitectura, urbanismo y transformaciones territoriales del Período Paracas en el Valle de Chincha, Boletin de Arqueología PUCP 17, 9-29.

Clarkson, P. B.

1990 The archaeology of the Nazca people: Environmental and cultural parameters, en: A. F. Aveni (ed.), The Lines of Nazca, 115-172, The American Philosophical Society, Philadelphia.

Cook, A. G.

1999 Asentamientos paracas en el valle bajo de Ica, Gaceta Arqueológica Andina 25, 61-90.

DeLeonardis, L.

2005 Early Paracas cultural contexts: New evidence from Callango, Andean Past 7, 27-55.

2012 Interpreting the Paracas body and its value in ancient Peru, en: J. K. Papadopoulos y G. Urton (ed.) The construction of value in the ancient world, 197-217, Cotsen Institute of Archaeology, University of California, Los Angeles. https://doi.org/10.2307/j.ctvdjrrxf.15

DeLeonardis, L. y M. D. Glascock

2013 From Queshqa to Callango: A Paracas obsidian assemblage from the lower Ica Valley, Peru, Nawpa Pacha 33, 163-192. https://doi.org/10.1179/0077629713Z.0000000008

DeLeonardis, L. y G. F. Lau

2004 Life, death and ancestors, en: H. Silverman (ed.), Andean Archaeology, 77-115, Blackwell Publishing, Oxford.

DeNiro, M. J.

1985 Postmortem preservation and alteration of in vivo bone collagen isotope ratios in relation to palaeodietary reconstruction, Nature 317, 806-809. https://doi.org/10.1038/317806a0

DeNiro, M. J. y S. Epstein

1981 Influence of diet on the distribution of nitrogen isotopes in animals, Geochimica et Cosmochimica Acta 45, 341-351. https://doi.org/10.1016/0016-7037(81)90244-1

DeNiro, M. J. y C. A. Hastorf

1985 Alteration of ${ }^{15} \mathrm{~N} /{ }^{14} \mathrm{~N}$ and ${ }^{13} \mathrm{C} /{ }^{12} \mathrm{C}$ ratios of plant matter during the initial stages of diagenesis: Studies utilizing archaeological specimens from Peru, Geochimica et Cosmochimica Acta 49, 97-115. https://doi. org/10.1016/0016-7037(85)90194-2

Engel, F. A.

1981 Prehistoric andean ecology. Man, settlement and the environment in the Andes. Stone tool typology, Hunter College, New York.

1991 Un desierto en tiempos prehispanicos, Universidad Nacional Agraria del Perú, Lima.

Greilich, S. y G. A. Wagner

2009 Light thrown on history - The dating of stone surfaces at the geoglyphs of Palpa using optically stimulated luminescence, en: M. Reindel y G. A. Wagner (eds.), New Technologies for Archaeology: Multidisciplinary Investigations in Palpa and Nasca, Peru, 271-186, Springer Verlag, Berlin. https://doi.org/10.1007/978-3540-87438-6_16

Hedges, R. E. M., J. G. Clement, C. D. L. Thomas y T. C. O’Connell

2007 Collagen turnover in the adult femoral mid-shaft: Modeled from anthropogenic radiocarbon tracer measurements, American Journal of Physical Anthropology 133, 808-816. https://doi.org/10.1002/ajpa.20598

Hillson, $S$.

2002 Dental anthropology, Cambridge University Press, Cambridge.

Hogg, A., J. Palmer, G. Boswijk, P. Reimer y D. Brown

2009 Investigating the inter hemispheric $14 \mathrm{C}$ offset in the 1st Millennium AD and assessment of laboratory bias and calibration errors, Radiocarbon 51, 1177-1186. https://doi.org/10.1017/S0033822200034238 
Isla, J.

2009 From hunters to regional lords: Funerary practices in Palpa, Peru, en: M. Reindel y G. A. Wagner (eds.), New Technologies for Archaeology: Multidisciplinary Investigations in Palpa and Nasca, Peru, 119-139, Springer Verlag, Berlin.

Lee-Thorp, J. A.

2008 On isotopes and old bone, Archaeometry50,925-950.https://doi.org/10.1111/j.1475-4754.2008.00441.x

Longin, R.

1971 New method of collagen extraction for radiocarbon dating, Nature 230, 241-242. https:/doi.org/ $10.1038 / 230241 \mathrm{a} 0$

Lovejoy, C.O., R. S. Meindl, R. P. Mensforth y T. J. Barton

1985 Multifactorial determination of skeletal age at death: a method and blind tests of its accuracy, American Journal of Physical Anthropology 68, 1-14. https://doi.org/10.1002/ajpa.1330680102

Lumbreras, L. G.

1974 The people and cultures of ancient Peru [traducción de B. Meggers], The Smithsonian Institution Press, Washington, D.C.

Massey, S. A.

1991 Social and political leadership in the lower Ica Valley, en A. Paul (ed.), Paracas art and architecture: Object and context in south coastal Peru, 315-348, University of Iowa Press, Iowa City.

Menzel, D., J. H. Rowe y L. E. Dawson

1964 The Paracas pottery of Ica: A study in style and time, University of California Press, Berkeley.

O'Connell, T. C. y R. E. M. Hedges

1999 Investigations into the effect of diet on modern human hair isotopic values, American Journal of Physical Anthropology 108, 409-425. https://doi.org/10.1002/(SICI)1096-8644(199904)108:4<409::AID$\mathrm{AJPA} 3>3.0 . \mathrm{CO} ; 2-\mathrm{E}$

O'Connell, T. C., R. E. M. Hedges, M. A. Healey y H. R. W. Simpson

2001 Isotopic comparison of hair, nail and bone: Modern analyses, Journal of Archaeological Science 28, 1247-1255. https://doi.org/10.1006/jasc.2001.0698

Pomeroy, E., J. T. Stock, S. R. Zakrzewski y M. M. Lahr

2010 A metric study of three types of artificial cranial modification from north-central Peru, International Journal of Osteoarchaeology 20, 317-334. https://doi.org/10.1002/oa.1044

Renneberg, R., S. Hummel y B. Herrmann

2009 The Nasca and their dear creatures - molecular genetic analysis of Pre-Coumbian camelid bones and textiles, en: M. Reindel y G. A. Wagner (eds.), New Technologies for Archaeology: Multidisciplinary Investigations in Palpa and Nasca, Peru, 193-206, Springer-Verlag, Berlin. https://doi.org/10.1007/978-3540-87438-6_12

Reinhard J.

1993 The Nazca lines: A New perspective on their origin and meaning, Editorial Los Pinos E.I.R.L, Lima.

Richards, M. P. y R. E. M. Hedges

1999 Stable isotope evidence for similarities in the types of marine foods used by late Mesolithic humans at sites along the Atlantic coast of Europe, Journal of Archaeological Science 26, 717-722. https://doi.org/ 10.1006/jasc.1998.0387

Reimer, P. J., E. Bard, A. Bayliss, J. W. Beck, P. G. Blackwell, C. Bronk Ramsey, P. M. Grootes, T. P. Guilderson, H. Haflidason, I. Hajdas, C. Hatt, T. J. Heaton, D. L. Hoffmann, A. G. Hogg, K. A. Hughen, K. F. Kaiser, B. Kromer, S. W. Manning, M. Niu, R. W. Reimer, D. A. Richards, E. M. Scott, J. R. Southon, R. A. Staff, C. S. M. Turney y J. van der Plicht

2013 IntCal13 and Marine13 radiocarbon age calibration curves 0-50,000 years cal BP, Radiocarbon 55, 1869-1887. https://doi.org/10.2458/azu_js_rc.55.16947 


\section{Rummel, S., S. Hölzl y P. Horn}

2007 Isotopensignaturen von Bio- und Geo-Elementen in der Forensik, en: B. Herrmann y K.-S. Saternus (ed.), Biologische Spurenkunde, Bd. 1 Kriminalbiologie, 381-407, Springer, Berlin. https://doi. org/10.1007/978-3-540-71111-7_18

Saitoh, M., M. Uzuka y M. Sakamoto

1969 Rate of hair growth, en: W. Montagna y R. L. Dobson (eds.), Advances in biology of skin; hair growth, 183-201, Pergamon Press, Oxford.

Scheuer, L. y S. Black

2000 Developmental juvenile osteology, Academic Press, London. https://doi.org/10.1016/ B978-012624000-9/50004-6

Schoeninger, M. J. y M. J. DeNiro

1984 Nitrogen and carbon isotopic composition of bone collagen from marine and terrestrial animals, Geochimica et Cosmochimica Acta 48, 625-639. https://doi.org/10.1016/0016-7037(84)90091-7

Silverman, H. I.

1990 Beyond the pampa: the geoglyphs in the valleys of Nazca, National Geographic Research 6, 435-456.

1996 The Formative period on the south coast of Peru: A critical review, Journal of World Prehistory 10, 95-146. https://doi.org/10.1007/BF02221074

2002a Ancient Nasca settlement and society, University of Iowa Press, Iowa City.

2002b Nasca settlement and society on the hundredth anniversary of Uhle's discovery of the Nasca style, en: W. H. Isbell y H. I. Silverman (ed.) Andean Archaeology I: Variations in Sociopolitical Organization, Springer New York. https://doi.org/10.1007/978-1-4615-0639-3_5

Stanish, C., H. Tantaleán, B. T. Nigra y L. Griffin

2014 A 2,300-year-old architectural and astronomical complex in the Chincha Valley, Peru, Proceedings of the National Academy of Sciences 111, 7218-7223. https://doi.org/10.1073/pnas.1406501111

Stanish, C. y H. Tantaleán,

2018 The Chincha Lines, Nawpa Pacha 38, 77-107. https://doi.org/10.1080/00776297.2018.1444949

Stone-Miller, R.

1992 To weave for the sun: Ancient Andean textiles in the Museum of Fine Arts, Boston, Thames and Hudson, London.

Tantaleán, H., C. Stanish, M. Zegarra y B. Nigral

2013 Paracas en el valle de Chincha: nuevos datos y explicaciones, Boletin de Arqueología PUCP 17, 31-56.

Tantaleán, H., C. Stanish, A. Rodríguez y K. Pérez

2016 The final days of Paracas in Cerro del Gentil, Chincha Valley, Peru, PLoS ONE 11, e0153465. https:// doi.org/10.1371/journal.pone.0153465

Thornton, E. K., S. D. Defrance, J. Krigbaum y P. R. Williams

2011 Isotopic evidence for Middle Horizon to 16th century camelid herding in the Osmore Valley, Peru, International Journal of Osteoarchaeology 211, 544-567. https://doi.org/10.1002/oa.1157

Tieszen, L. L. y M. Chapman

1992 Carbon and nitrogen isotopic status of the major marine and terrestrial resources in the Atacama Desert of northern Chile, Proceedings of the First World Congress on Mummy Studies, 409-426, Museo Arqueológico y Etnográfico de Tenerife, Tenerife.

Tripcevich, N. y D. A. Contreras

2011 Quarrying evidence at the Quispisisa obsidian source, Ayacucho, Peru, Latin American Antiquity 22, 121-136. https://doi.org/10.7183/1045-6635.22.1.121

Unkel, I., M. Reindel, H. Gorbahn, J. Isla, B. Kromer y V. Sossna

2012 A comprehensive numerical chronology for the pre-Columbian cultures of the Palpa valleys, south coast of Peru, Journal of Archaeological Science 39, 2294-2303. https://doi.org/10.1016/j.jas.2012.02.021 
Wallace, D. T.

1962 Cerrillos, an early Paracas site in Ica, Peru, American Antiquity 27, 303-314. https://doi. org/10.2307/277797

Webb, E., C. White y F. Longstaffe

2013 Dietary shifting in the Nasca region as inferred from the carbon- and nitrogen-isotope compositions of archaeological hair and bone, Journal of Archaeological Science 40, 129-139. https://doi.org/10.1016/j. jas.2012.08.020

White, C. D.

1993 Isotopic determination of seasonality in diet and death from Nubian mummy hair, Journal of Archaeological Science 20, 657-666. https://doi.org/10.1006/jasc.1993.1040

Williams, C.

1980 Arquitectura y urbanismo en el antiguo Perú, en: Historia del Perú, tomo VIII, Perú Republicano y procesos e instituciones, 466-468, Editorial Mejía Baca, Lima.

\section{Williams, J. S. y M. A. Katzenberg}

2012 Seasonal fluctuations in diet and death during the late horizon: a stable isotopic analysis of hair and nail from the central coast of Peru, Journal of Archaeological Science 39, 41-57. https://doi.org/10.1016/j. jas.2011.07.013 